\title{
Fréquenter les Gouttes de lait. L'expérience des mères montréalaises, 1910-1965
}

\section{Denyse Baillargeon}

Volume 50, numéro 1, été 1996

URI : https://id.erudit.org/iderudit/305488ar

DOI : https://doi.org/10.7202/305488ar

Aller au sommaire du numéro

\section{Éditeur(s)}

Institut d'histoire de l'Amérique française

\section{ISSN}

0035-2357 (imprimé)

1492-1383 (numérique)

Découvrir la revue

\section{Citer cet article}

Baillargeon, D. (1996). Fréquenter les Gouttes de lait. L'expérience des mères montréalaises, 1910-1965. Revue d'histoire de l'Amérique française, 50(1), 29-68. https://doi.org/10.7202/305488ar

\section{Résumé de l'article}

La lutte contre la mortalité infantile, qui s'est engagée au début du siècle un peu partout en Occident, s'est concrétisée par la mise en place de centres de distribution de lait sain, bientôt transformés en cliniques de puériculture où les mères venaient apprendre comment soigner leurs bébés. À Montréal, ces cliniques, appelées « Gouttes de lait » ont figuré au premier plan des services socio-sanitaires jusqu'au début des années 1970. À l'aide d'entrevues, mais aussi de sources documentaires et statistiques provenant principalement du Service de santé de la Ville de Montréal, cet article se propose de scruter les motivations des femmes qui ont fréquenté les Gouttes de lait montréalaises en distinguant deux périodes, soit 1910-1930 et 1930-1965. Ce découpage chronologique a été en partie déterminé par la nature des sources utilisées pour explorer chacune des périodes, mais il correspond également à des changements d'attitudes importants de la part des mères de famille face au processus de médicalisation de la petite enfance, car c'est à partir des années 1930 que les cliniques se sont véritablement imposées auprès de la population féminine. L'histoire des Gouttes de lait entre 1910 et 1930, de même que les témoignages recueillis, laissent voir cependant qu'il a fallu l'intervention de plusieurs facteurs, comme la baisse de la natalité et l'amélioration générale des conditions de vie, pour que les femmes adoptent finalement certaines des pratiques recommandées par les médecins, à commencer par la fréquentation plus régulière de la Goutte de lait.
Tous droits réservés @ Institut d'histoire de l'Amérique française, 1996
Ce document est protégé par la loi sur le droit d'auteur. L’utilisation des services d'Érudit (y compris la reproduction) est assujettie à sa politique d'utilisation que vous pouvez consulter en ligne.

https://apropos.erudit.org/fr/usagers/politique-dutilisation/ 


\title{
FRÉQUENTER LES GOUTTES DE LAIT L'EXPÉRIENCE DES MÈRES MONTRÉALAISES 1910-1965'
}

\author{
DENYSE BAILLARGEON \\ Département d'histoire \\ Université de Montréal
}

\section{RÉSUMÉ}

La lutte contre la mortalité infantile, qui s'est engagée au début du siècle un peu partout en Occident, s'est concrétisée par la mise en place de centres de distribution de lait sain, bientôt transformés en cliniques de puériculture où les mères venaient apprendre comment soigner leurs bébés. À Montréal, ces cliniques, appelées «Gouttes de lait» ont figuré au premier plan des services socio-sanitaires jusqu'au début des années 1970. Â l'aide d'entrevues, mais aussi de sources documentaires et statistiques provenant principalement du Service de santé de la Ville de Montréal, cet article se propose de scruter les motivations des femmes qui ont fréquenté les Gouttes de lait montréalaises en distinguant deux périodes, soit 1910-1930 et 1930-1965. Ce découpage chronologique a été en partie déterminé par la nature des sources utilisées pour explorer chacune des périodes, mais il correspond également à des changements d'attitudes importants de la part des mères de famille face au processus de médicalisation de la petite enfance, car c'est à partir des années 1930 que les cliniques se sont véritablement imposées auprès de la population féminine. L'histoire des Gouttes de lait entre 1910 et 1930 , de même que les témoignages recueillis, laissent voir cependant qu'il a fallu l'intervention de plusieurs facteurs, comme la baisse de la natalité et l'amélioration générale des conditions de vie, pour que les femmes adoptent finalement certaines des pratiques recommandées par les médecins, à commencer par la fréquentation plus régulière de la Goutte de lait.

\section{ABSTRACT}

The creation of milk distribution centres was a key measure in the battle against infant mortality that began early in the twentieth century in much of the West. The

1. Cette recherche a bénéficié du support financier de l'Institut Hannah d'Histoire de la médecine que je tiens à remercier. Mes plus sincères remerciements vont également à MarieJosée Blais, qui a agi à titre d'assistante de recherche durant toute la durée du projet et s'est révélée une précieuse collaboratrice, de même qu'à Michèle Dagenais et Othmar Keel qui ont bien voulu lire une première version de ce texte et me faire part de leurs commentaires. Merci enfin aux personnes qui ont évalué ce texte pour leurs précieuses suggestions. 
centres soon evolved into clinics where mothers could learn how to look after their babies. Known as "Gouttes de lait" in Montréal, these Well Baby Clinics were important among the city's socio-sanitary services until the beginning of the 1970s. The article draws on interviews as well as documents and statistics originating principally in the City of Montréal Health Service in order to examine the motivations of the women who used the Gouttes de lait over two periods, 1910 to 1930 and 1930 to 1965 . While this periodization reflects the varying sources used to study the earlier and the later years, it also corresponds to important changes in mothers' attitudes towards the increasing medicalization of early childhood: the clinics only became popular among the female population beginning in the 1930s. However, the history of the Gouttes de lait from 1910 to 1930 , as well as the testimony gathered in the interviews, shows that several factors - notably, the lower birthrate and the general improvement in living conditions - help explain why women ultimately adopted some of the practices recommended by physicians, including more regular use of the Gouttes de lait.

Au début du siècle, Montréal comptait parmi les villes occidentales les plus meurtrières pour les nouveau-nés. Davantage qu'à Londres, Paris, New York ou Toronto, les enfants y mouraient avant d'avoir atteint l'âge d'un an, surtout de diarrhées. Au sein même de la population montréalaise, les familles canadiennes-françaises étaient les plus touchées. Ainsi, en 1910, alors que chez les anglo-protestants, le taux de mortalité infantile se situait à 163 pour mille, chez les franco-catholiques, cette proportion atteignait les 224 pour mille, contre 207 pour mille chez les catholiques d'autres origines et 94 pour mille parmi la population juive ${ }^{2}$. Comme dans la plupart des grandes agglomérations urbaines des pays industrialisés, des facteurs économiques et environnementaux - notamment le faible niveau de vie des familles ouvrières, l'insalubrité générale des quartiers où elles habitaient et la piètre qualité du lait, dont la pasteurisation ne devint obligatoire qu'en 1926 - étaient en bonne partie responsables de ce phénomène. Il est cependant évident que la forte mortalité des nouveau-nés francophones contribuait à faire grimper les taux montréalais à des niveaux particulièrement catastrophiques, ce qui laisse supposer qu'en plus des inégalités sociales et des mauvaises conditions sanitaires, certains comportements culturels pouvaient influer sur les chances de survie des nourrissons. À cet égard, plusieurs observateurs de l'époque ont noté que les mères canadiennesfrançaises allaitaient leurs enfants moins longtemps que les autres, un fait que semblent accréditer les travaux de Thornton, Olson et Thuy Thach sur la mortalité infantile à Montréal au XIX ${ }^{e}$ siècle ${ }^{3}$. Dans l'état

2. Montréal, Rapport sur l'état sanitaire de la cité de Montréal (ci-après, RASS) (1912), 58.

3. Patricia Thornton, Sherry Olson et Quoc Thuy Thach, «Dimensions sociales de la mortalité infantile à Montréal au milieu du $\mathrm{XIX}^{\circ}$ siècle», Annales de démographie historique (1988): 299-325. 
des recherches actuelles, il est difficile d'expliquer ce comportement, mais il $\mathrm{y}$ a tout lieu de croire que le sevrage précoce était aussi à l'origine d'un plus grand nombre de grossesses chez les Canadiennes françaises, les naissances rapprochées entraînant à leur tour l'épuisement de la mère et des risques supplémentaires pour la vie du nouveau-né.

La mortalité infantile n'était certes pas une réalité nouvelle au début du $\mathrm{XX}^{\mathrm{e}}$ siècle, mais c'est à partir de cette époque que l'on a tenté d'y faire échec, du moins en Occident. Le contexte socio-économique et politique était en effet propice à l'émergence de graves inquiétudes au sujet de l'avenir des nations industrialisées, ce qui a favorisé la montée d'un vaste mouvement en faveur de la sauvegarde de l'enfance. Dans chacun des pays en cause, des événements ou des phénomènes particuliers ont sonné l'alarme, mais, partout, la question nationale était au centre des débats. En Angleterre, par exemple, le recrutement de soldats lors de la guerre des Boers avait révélé l'état de santé déplorable de la population ouvrière et fait craindre pour la suprématie de l'Empire britannique. En France, la baisse de la natalité, alors que l'Allemagne se faisait toujours menaçante, en vint à être considérée comme un véritable drame national. Aux États-Unis, comme au Canada anglais, on craignait surtout de voir les populations blanches être submergées par des vagues d'immigrants provenant d'Europe centrale et méridionale ${ }^{4}$. En ce qui concerne le Québec, il ne s'agissait pas tant de défendre un empire que d'assurer la «survivance de la race». Les élites laïques et religieuses francophones, traumatisées

4. Pour une perspective comparative au sujet de la lutte contre la mortalité infantile dans les pays européens et plus généralement du mouvement en faveur du bien-être infantile et maternel, voir Seth Koven et Sonya Michel, «Womanly Duties: Maternalist Politics and the Origins of Welfare States in France, Germany, Great Britain, and the United States, 1880-1920», American Historical Review, 95,4 (octobre 1990): 1076-1114; Seth Koven et Sonya Michel, dir., Mothers of a New World. Maternalist Politics and the Origins of Welfare States (New York et Londres, Routledge, 1993); Gisela Bock et Pat Thane, dir., Maternity and Gender Policies. Women and the Rise of the European Welfare States 1880s-1950s (Londres et New York, Routhledge, 1994), et Susan Pedersen, Family, Dependance, and the Origins of the Welfare State. Britain and France 1914-1945 (Cambridge, Cambridge University Press, 1993). Sur la situation américaine, voir Molly Ladd-Taylor, Mother Work. Women, Child Welfare, and the State, 1890-1930 (Chicago, University of Illinois Press, 1994). Pour le Canada anglais, voir en particulier Cynthia R. Abeele, «'The Infant Soldier': the Great War and the Medical Campaign for Child Welfare», Canadian Bulletin of History of Medicine/Bulletin canadien d'histoire de la médecine (CBMH/ BCHM), 5,2 (hiver 1988): 99-119; Cynthia R. Comacchio, Nations Are Built of Babies. Saving Ontario's Mothers and Children 1900-1940 (Montréal, McGill-Queen's University Press, 1993); Meryn Stuart, "Let Not the People Perish for Lack of Knowledge»: Public Health Nursing and the Ontario Rural Child Welfare Project, 1916-1930, thèse de doctorat (Nursing), University of Pennsylvania, 1987, et «Ideology and Experience: Public Health Nursing and the Ontario Rural Child Welfare Project, 1920-1925», CBMH/BCHM, 6,2 (hiver 1989): 111-131. 
par l'émigration des Canadiens français aux États-Unis et par le sort réservé aux leurs à l'intérieur même du Canada, considéraient en effet la mortalité infantile comme une véritable menace pour la survie de la nation française en Amérique ${ }^{5}$. Ici comme ailleurs, ces préoccupations à fortes consonances nationalistes débouchèrent sur une volonté nouvelle de préserver la vie des nouveau-nés, capital humain essentiel au «progrès, [à] l'existence, et [à] la suprématie des diverses collectivités $^{6} »$. Pour y arriver, on comptait sur les récents développements de la médecine, en particulier les découvertes en microbiologie et en bactériologie, qui permettaient de mieux comprendre les causes de certaines maladies et de proposer des moyens à prendre pour les prévenir, à défaut de pouvoir les guérir.

À peu près partout où il s'est enraciné, le mouvement de lutte contre la mortalité infantile a d'abord été initié par des réformateurs sociaux, le plus souvent des groupes de femmes, travaillant en collaboration avec des médecins et parfois des membres du clergé, avant d'être finalement pris en charge par les autorités en santé publique. Dans un premier temps, ces groupes réformistes mirent en place des centres de distribution de lait sain, mais, assez rapidement, ces derniers furent transformés en cliniques de puériculture visant à enseigner aux mères comment soigner leurs bébés; au cours de cette seconde phase, les médecins prirent la direction des opérations et des infirmières vinrent remplacer les bénévoles féminines.

À Montréal, c'est en 1910 que les trois premiers centres de distribution de lait furent mis sur pied sur une base permanente, soit à peu près en même temps qu'à Toronto ${ }^{7}$. Le premier provenait de l'initiative de réformateurs sociaux et de féministes anglophones et entendait desservir l'ensemble de la population pauvre de cette communauté linguistique. Le second, rattaché à l'hôpital Sainte-Justine pour enfants, fut fondé par des dirigeantes de la Fédération nationale Saint-Jean-Baptiste (FNSJB), également responsables de la création de l'hôpital. Le troisième, affilié à la paroisse ouvrière Saint-EnfantJésus, était l'œuvre du docteur Séverin Lachapelle, un spécialiste des soins infantiles, et du curé LePailleur, qui s'étaient rendus en France pour examiner le fonctionnement des Gouttes de lait, mises sur pied

5. Voir par exemple les propos du docteur Eugène Gagnon dans «Comment diminuer la mortalité infantile (1)», Union médicale du Canada (UMC), 34,12 (décembre 1910): 714.

6. RASS (1913): xiii.

7. Dans cette ville, le premier dépôt de lait fut inauguré en 1912 (Cynthia R. Abeele, «'The Infant Soldier'», 109). À Montréal, le premier dépôt du même genre remonte à 1901, mais il ne fut en opération que quelques mois. 
à partir de la fin du XIX siècle $^{8}$. Le Service de santé de la Ville fournissait une partie de leur financement, mais chacun de ces centres était administré localement.

En quelques années, sous l'impulsion de petits groupes de réformistes, une vingtaine de centres similaires furent créés, dont plus d'une quinzaine rattachés à des paroisses catholiques francophones situées dans les quartiers les plus pauvres de la ville?. Dès le départ, les centres francophones se sont distingués par leur appellation d'origine française (Gouttes de lait) et par un encadrement paroissial qui en faisait des institutions confessionnelles. Leur fonctionnement était assuré grâce à une collaboration, parfois malaisée, entre médecins et dames patronnesses, ces dernières étant le plus souvent des membres de la FNSJB. En fait, à partir de 1914, la FNSJB contribua activement à l'organisation ou à la réorganisation d'une quinzaine de ces centres, que la Ville voulait voir transformés en cliniques de puériculture. À la suite d'un conflit avec les médecins, survenu en 1916, la FNSJB fut cependant écartée de l'organisation des Gouttes de lait paroissiales ${ }^{10}$, tandis qu'en 1919, le Service de santé, maintenant doté d'un service d'hygiène infantile, décidait de créer son propre réseau municipal de consultations ${ }^{11}$. Mais contrairement à ce qui s'est produit à Toronto, où la Ville a pris en charge l'ensemble des cliniques créées par les philanthropes au lendemain de la Grande Guerre ${ }^{12}$, à Montréal, les autorités en santé publique considéraient qu'elles pouvaient difficilement faire abstraction des cliniques privées qui bénéficiaient de l'appui du clergé. Les cliniques municipales furent donc ouvertes uniquement dans les paroisses où il n'existait pas de Goutte de lait, et le directeur du Service de santé s'assura d'obtenir l'approbation de

8. $\quad \mathrm{D}^{\mathrm{r}}$ Joseph Gauvreau, L'Oeuvre des Gouttes de lait paroissiales (Montréal, École sociale populaire (ESP), l'Oeuvre des tracts, $\mathrm{n}^{\circ} 198$, décembre 1935 et $\mathrm{D}^{\mathrm{r}} \mathrm{J}$. E. Dubé, «Les débuts de la lutte contre la mortalité infantile à Montréal. Fondation de la première Goutte de lait», UMC, 65 (1936): 879-891, 986-993 et 1088-1102.

9. RASS (1918-1919), 41.

10. L'analyse du conflit qui a opposé la FNSJB et les médecins, de même que ceux qui sont survenus entre ces derniers et la Ville dépasse le cadre de cet article. Nous avons l'intention de nous y attarder dans «Gouttes de lait et soif de pouvoir: les dessous de la lutte contre la mortalité infantile à Montréal, 1910-1953» (à paraître).

11. Adélard Groulx, «La division de l'hygiène de l'enfance au Service de santé de la Ville de Montréal. Son histoire et son programme actuel», $U M C, 79,11$ (novembre 1950): 274275. Selon François Guérard, c'est après la Première Guerre mondiale que les autorités provinciales commencèrent à s'intéresser de plus près à la santé publique et à considérer «qu'il appartenait à l'État de prendre en charge et de coordonner les interventions d'hygiène publique» (Guérard, «L'hygiène publique au Québec de 1887 à 1939: centralisation, normalisation et médicalisation», communication présentée dans le cadre du congrès de la Société historique du Canada, Montréal, août 1995, 14).

12. Cynthia R. Abeele, «'The Infant Soldier'», 114. 
l'archevêque de Montréal avant de procéder ${ }^{13}$. Même si la Ville n'a jamais distribué de lait dans ses consultations et que les associations privées abandonnèrent graduellement cette pratique, le terme «Goutte de lait» avait eu le temps de s'imposer au sein de la population et devait rester accolé à toutes les cliniques de puériculture de la ville.

Au début des années 1930, 68 cliniques étaient en opération à Montréal; 36 étaient contrôlées par le Service de santé de la Ville et 32 par des organismes privés, dont 18 par des associations paroissiales francophones ${ }^{14}$. Cette triple structure allait perdurer jusqu'en 1953, alors que la Ville prenait le contrôle de l'ensemble du réseau des Gouttes de lait paroissiales privées ${ }^{15}$. Malgré l'existence de trois réseaux parallèles, une particularité bien montréalaise, et de la longévité exceptionnelle des Gouttes de lait privées, les cliniques fondées à Montréal au début du siècle, poursuivaient les mêmes objectifs que celles qui existaient un peu partout en Europe et en Amérique du Nord vers la même époque. Essentiellement, il s'agissait d'enseigner aux mères des notions élémentaires d'hygiène et de les conseiller dans l'art de soigner les bébés. La promotion de l'allaitement maternel représentait l'article numéro un de ce programme d'éducation populaire, mais on voulait également amener les femmes à abandonner certaines pratiques jugées nuisibles pour la santé des enfants. Pour ce faire, il fallait convaincre les mères d'amener régulièrement leurs nouveau-nés à la consultation afin qu'ils soient examinés et pesés et afin de recevoir des directives du médecin et de l'infirmière.

Les médecins et les autorités en santé publique admettaient en effet que la pauvreté était en partie responsable de la forte mortalité chez les jeunes enfants, mais, dans l'ensemble, ils considéraient que l'ignorance et la négligence des femmes étaient des causes bien plus directes et généralisées: «La misère en certains milieux peut [...] entrer en ligne de compte, mais il ne faut pas oublier que l'ignorance des mères plus encore que la misère est responsable des erreurs commises dans l'alimentation ${ }^{16} . »$ En refusant de considérer sérieusement l'impact de la pauvreté sur la santé des nourrissons, ils évitaient de

13. Archives de la Ville de Montréal, $3^{e}$ série, Lettre du docteur $\mathrm{S}$. Boucher, directeur du Service de santé de la Ville de Montréal, à $\mathrm{M}^{\mathrm{gr}}$ Georges Gauthier, archevêque coadjuteur de Montréal, 27 mai 1929 et Lettre du docteur Boucher à Jules Crépeau, Directeur des Services de la Ville, 2 juillet 1929.

14. RASS (1930), 113.

15. On comptait alors 63 cliniques municipales, francophones et anglophones, tandis que six consultations anglophones indépendantes étaient toujours en opération. RASS (1953), 43, et 1954-1955-1956, 57.

16. $\mathrm{D}^{r}$ Eugène Gagnon, «Comment diminuer la mortalité infantile (1)», 716. Le même genre de propos se retrouve dans le rapport du Service de santé de la Ville de Montréal pour l'année 1913: «si la pauvreté (est) un des trois plus grands facteurs de la mortalité infantile, l'ignorance et la négligence, en (sont) deux autres aussi malfaisants» (xiii). 
s'interroger sur l'efficacité réelle du type d'intervention qu'ils préconisaient et ils esquivaient la question de l'accessibilité des soins de santé pour les plus démunis. Cette façon de concevoir le problème de la mortalité infantile, attribué essentiellement aux mères, et sa solution, l'éducation de ces dernières, visait en fait à maintenir un certain équilibre entre, d'une part, la nécessité de faire face à un problème national et humanitaire et, d'autre part, celle de protéger les intérêts économiques et professionnels des praticiens qui se sentaient menacés par l'ouverture de ces cliniques: pour reprendre les propos du docteur DeGrandpré, il s'agissait de «soigner les bébés sans faire mourir les médecins ${ }^{17}{ }^{»}$. Les fonctions du médecin de la Goutte de lait, qu'elle soit privée ou municipale, étaient donc strictement définies afin de ne pas faire concurrence à la pratique privée, les mères ne pouvant y recevoir que des conseils sur l'alimentation de leurs enfants, et ces derniers, des traitements reliés à des troubles du système digestif ${ }^{18}$.

L'intérêt des réformateurs sociaux et des autorités publiques pour la santé des nourrissons a déjà fait l'objet de nombreux débats dans l'historiographie féministe. Leurs motivations, en particulier celles des médecins qui ont souvent été des figures dominantes à l'intérieur de ce groupe, ont été scrutées, les différentes mesures qu'ils proposaient et qui ont été mises en œuvre, examinées sous plusieurs angles et les conséquences de leurs interventions pour les mères, soupesées ${ }^{19}$.

17. «Compte rendu de la première convention des Gouttes de lait de Montréal», UMC, 42,6 (juin 1913): 327. Pour une discussion de la position et des intérêts des médecins en rapport avec la lutte contre la mortalité infantile, voir Comacchio, Nations Are Built of Babies, en particulier le premier chapitre.

18. Gouttes de lait de Montréal. Section française. Constitution et règlements (Montréal, Bureau municipal d'hygiène et de la statistique, 1915), 13.

19. En plus des titres mentionnés à la note 4, voir Anna Davin, «Imperialism and Motherhood», History Workshop, 5, (printemps 1978): 9-65; Jane Lewis, The Politics of Motherhood. Child and Maternal Welfare in England, 1900-1939 (Montréal, McGill-Queen's University Press, 1980); Ellen Ross, Love and Toil. Motherhood in Outcast London, 1870-1918 (Oxford, Oxford University Press, 1993); Deborah Dwork, War Is Good for Babies and Other Young Children. A History of the Infant and Child Welfare Movement in England, 1898-1918 (London, Tavistock, 1987); Veronica Strong-Boag, «Intruders in the Nursery: Childcare Professionals Reshape the Years One to Five, 1920-1940», dans Joy Parr, dir., Childhood and Family in Canadian History (Toronto, McClelland and Stewart, 1982), 160-177; Suzanne Buckley, «Ladies or Midwifes? Efforts to Reduce Infant and Maternal Mortality», dans L. Kealy, dir., A not Unreasonable Claim: Women and Reform in Canada, 1880-1920 (Toronto, Women's Press, 1979), 131-149; Dianne Dodd, «Advice to Parents: the Blue Books, Helen MacMurchy, MD, and the Federal Department of Health, 1920-34», CBMH/BCHM, 8,2 (hiver 1991): 203230; Katherine Arnup, «Educating Mothers: Government Advice for Women in the Inter-War Years», dans Katherine Arnup, Andrée Lévesque et Ruth Roach Pierson, dir., Delivering Motherhood. Maternal Ideologies and Practices in the 19th and 20th Centuries (Londres, Routhledge, 1990), 190-210, et Education for Motherhood. Advice for Mothers in TwentiethCentury Canada (Toronto, University of Toronto Press, 1994); Claudine Pierre-Deschesne, «Santé publique et organisation de la profession médicale au Québec, 1870-1918», Revue d'histoire de l'Amérique française, 35,3 (décembre 1981): 335-375. 
Orchestrées par des médecins, mais supportées également par des femmes regroupées au sein d'associations féministes et philanthropiques, et appuyées par certains éléments du clergé et par les autorités en santé publique, les campagnes de lutte contre la mortalité infantile soulèvent en effet des questions fondamentales à propos de la construction sociale de la maternité, car, à travers cette lutte pour assurer la survie des nourrissons, c'est l'ensemble des responsabilités maternelles que ces propagandistes cherchaient à redéfinir. La «bonne mère» est devenue celle qui se préoccupait de la santé de ses enfants et qui acceptait d'abandonner les méthodes et les recettes traditionnelles en matière de soins infantiles pour suivre les directives d'un médecin ou d'une infirmière agissant sous ses ordres ${ }^{20}$. De manière générale, toutes les femmes étaient visées par ce discours prescriptif, mais il est évident que les plus pauvres étaient plus particulièrement ciblées. Celles qui faisaient partie des classes plus aisées ont sans doute intégré plus rapidement ces nouveaux comportements qui s'accordaient avec leur mode de vie et leurs valeurs; plusieurs d'entre elles ont d'ailleurs activement participé à leur diffusion.

Tout au long du $\mathrm{XX}^{\mathrm{e}}$ siècle, de plus en plus de mères des classes populaires ont été convaincues de la nécessité d'assurer un suivi médical pour leurs nouveau-nés. Les progrès enregistrés dans la fréquentation des Gouttes de lait, surtout à partir des années 1920, attestent cette tendance. On peut toutefois se demander si l'augmentation des taux de fréquentation peut s'interpréter uniquement comme une manifestation de l'emprise des médecins sur les mères. Comme certaines historiennes l'ont déjà souligné, on peut à tout le moins présumer que les femmes ont également joué un rôle important dans la redéfinition de leurs responsabilités de soignante et d'éducatrice ${ }^{21}$, ce qui implique que ce processus de médicalisation de la petite enfance n'a pu se faire sans leur accord. Reste à savoir dans quelle mesure, pour quelles raisons et à quelles conditions elles ont accepté de s'en remettre à des spécialistes pour les guider dans les soins à apporter à leurs enfants.

20. Ce discours était encore présent au moment de la Deuxième Guerre mondiale, comme en témoigne un texte du $\mathrm{D}^{\mathrm{r}}$ Adrien Plouffe publié dans le Bulletin d'Hygiène de la Ville de Montréal en 1944. On peut y lire «Maman de la province de Québec, [...] ayez une sorte de culte pour le médecin et vous mettrez de votre côté toutes les chances que vous avez de garder vos bébés en bonne santé et vivants» («Ce que nous devons faire pour sauver nos bébés», Bulletin d'hygiène de la Ville de Montréal, 30,3 (mai-juin 1944): 10).

21. Voir Comacchio, Nations Are Built of Babies; Lewis, The Politics of Motherhood et «Motherhood Issues in the Late Nineteenth and Twentieth Centuries», dans Arnup et al., dir., Delivering Motherhood, 1-19; Dodd, «Advice to Parents». 
À l'aide d'entrevues, mais aussi de sources documentaires et statistiques provenant principalement du Service de santé de la Ville de Montréal, cet article se propose de scruter la question des motivations des femmes qui ont fréquenté les Gouttes de lait montréalaises en distinguant deux périodes, soit 1910-1930 et 1930-1965. Ce découpage chronologique a été en partie déterminé par la nature des sources utilisées pour explorer chacune des périodes, mais il correspond également à des changements d'attitudes importants de la part des mères de famille face au processus de médicalisation. C'est en effet à partir des années 1930 que les cliniques se sont véritablement imposées auprès des mères montréalaises. L'histoire des Gouttes de lait entre 1910 et 1930, de même que les témoignages recueillis, laissent voir cependant qu'il a fallu l'intervention de plusieurs facteurs, comme la baisse de la natalité et l'amélioration générale des conditions de vie, pour que les femmes adoptent finalement certaines des pratiques recommandées par les médecins, à commencer par la fréquentation plus régulière de la Goutte de lait.

\section{FRÉQUENTER LES GOUTTES DE LAIT ENTRE 1910 ET 1930}

Comment les femmes ont-elles réagi face à ce nouveau service durant ses premières années d'existence? Pourquoi et comment l'ontelles utilisé? Dans quelle mesure répondait-il à leurs besoins? À défaut de pouvoir s'en remettre à leurs témoignages, certaines données statistiques, de même que les rapports annuels du Service de santé de la Ville de Montréal et les procès-verbaux de la Commission centrale de la Goutte de lait, affiliée à la FNSJB, permettent de lever un coin du voile sur ces questions.

Mentionnons tout d'abord que la proportion exacte des nouveaunés qui étaient amenés à la Goutte de lait par rapport aux naissances annuelles dans les paroisses desservies ou dans l'ensemble de la ville est presque impossible à établir pour la période 1910-1924, parce que les enfants pouvaient être comptabilisés plusieurs fois au cours d'une même année ${ }^{22}$. Après 1924, la Ville commença à distinguer les nouveaux inscrits du total des bébés amenés à la consultation dans les statistiques qu'elle colligeait pour l'ensemble du réseau, mais d'autres problèmes méthodologiques se posent du fait que les enfants pouvaient être admis jusqu'à l'âge de deux ans. De plus, au début de chaque nouvelle année, le nom des enfants qui étaient toujours inscrits sur les listes à la fin de l'année précédente étaient reportés sur les

22. En effet, lorsqu'ils n'étaient pas amenés à la Goutte de lait durant plus d'un mois, ils étaient radiés des listes pour être réinscrits à nouveau s'ils se présentaient plus tard, une situation assez courante si on en croit les doléances des responsables. ("Fête de la Goutte de lait», Bulletin paroissial Immaculée-Conception, 18,3 (janvier 1927): 10, et RASS (1922), 83). 
nouvelles listes et comptaient comme de nouvelles inscriptions pour l'année en cours. Les données disponibles montrent cependant que le nombre des inscriptions aux différentes Gouttes de lait n'a cessé d'augmenter au cours des années 1910 et 1920, alors que les naissances restaient à peu près stables. On peut donc en conclure que de plus en plus de mères amenaient leurs enfants à la consultation, sans pouvoir préciser avec exactitude dans quelle proportion pour chaque année. Le tableau suivant, présenté à titre indicatif, donne un aperçu de la progression du nombre des cliniques et des inscriptions, de même que le total des naissances pour le territoire de la Ville de Montréal entre 1915 et 1930.

TABLEAU 1

Inscriptions aux Gouttes de lait privées et aux consultations municipales et nombre annuel des naissances pour Montréal $1915-1930$

\begin{tabular}{|c|c|c|c|c|c|c|}
\hline Années & $\begin{array}{c}\text { Nombre } \\
\text { total de } \\
\text { cliniques }\end{array}$ & $\begin{array}{c}\text { Inscriptions } \\
\text { aux Gouttes } \\
\text { de lait privées } \\
\text { francophones }\end{array}$ & $\begin{array}{c}\text { Inscriptions } \\
\text { aux Goutles } \\
\text { de lait privées } \\
\text { anglophones }\end{array}$ & $\begin{array}{c}\text { Inscriptions } \\
\text { aux } \\
\text { consultations } \\
\text { municipales }\end{array}$ & $\begin{array}{c}\text { Total des } \\
\text { inscriptions }\end{array}$ & $\begin{array}{c}\text { Total des } \\
\text { naissances }\end{array}$ \\
\hline 1915 & 28 & nd & nd & nil & 3673 & 20692 \\
1916 & 25 & nd & nd & nd & 3203 & 19759 \\
1917 & 27 & $n d$ & nd & nd & 3835 & 19664 \\
1918 & 28 & $n d$ & nd & $n d$ & 5018 & 20373 \\
1919 & 39 & 3681 & 1312 & 2129 & 7122 & 19959 \\
1920 & 43 & 4028 & 1525 & 3387 & 8940 & 21 \\
1921 & 43 & 4768 & 1778 & 3783 & 10329 & 21136 \\
1922 & 44 & 5067 & 2523 & 4517 & 12107 & 20780 \\
1923 & 46 & 4989 & 3001 & 4005 & 11995 & 19933 \\
1924 & 47 & 3709 & 1899 & 3008 & 8616 & 21500 \\
1925 & 53 & 4061 & 3031 & 2998 & 10090 & 21976 \\
1926 & 55 & 3975 & 2854 & 2780 & 9609 & 19650 \\
1927 & 50 & 3799 & 2828 & 2112 & 8739 & 19458 \\
1928 & 57 & 4132 & 2504 & 3419 & 10055 & 20252 \\
1929 & 71 & 4023 & 2359 & 5312 & 11694 & 20622 \\
1930 & 68 & 6325 & 2325 & 5644 & 14294 & 21044 \\
\hline
\end{tabular}

Sources: Rapport sur l'état sanitaire de la cité de Montréal (1915-1930) et Québec, Annuaire statistique (1915-1930).

Selon ce tableau, deux enfants sur trois environ auraient fréquenté les cliniques de la Goutte de lait à Montréal au début des années 1930. Le service était certainement devenu accessible à une plus forte proportion de la population, puisque la ville avait ouvert 20 nouvelles cliniques en 1929, mais, compte tenu des méthodes comptables discutées plus haut, la proportion réelle des bébés inscrits par rapport au total des naissances était très certainement moindre; en fait, en additionnant le nombre des naissances pour l'année 1929 et 1930 et 
en divisant le total des inscriptions pour 1930 par ce nombre, on arrive à un pourcentage de $34 \%$, un chiffre probablement plus près de la réalité, puisque des enfants nés en 1929 sont compris dans le nombre des inscriptions pour $1930^{23}$. Il s'agit tout de même là d'une proportion respectable si on tient compte du fait que ces cliniques ne s'adressaient qu'à une portion de la population qui ne pouvait recourir aux services privés d'un médecin ${ }^{24}$.

Par ailleurs, les statistiques mensuelles, établies par la Ville pour ses propres cliniques à partir de 1920 , montrent que le nombre des fréquentations augmentait de manière très sensible durant les mois d'été et ce, pour toute la période où ce genre de données ont été recueillies.

TABLEAU 2

Nombre des inscriptions mensuelles aux consultations municipales $1920-1936$

\begin{tabular}{||c|c|c|c|c|c|c||}
\hline Années & $\begin{array}{c}\text { Janvier- } \\
\text { février }\end{array}$ & Mars-avril & Mai-juin & Juillet-août & $\begin{array}{c}\text { Septembre- } \\
\text { octobre }\end{array}$ & $\begin{array}{c}\text { Novembre- } \\
\text { décembre }\end{array}$ \\
\hline 1920 & 375 & 241 & 423 & 1669 & 225 & nd \\
1921 & 804 & 470 & 1057 & 1206 & 436 & 281 \\
1922 & 1027 & 480 & 784 & 1505 & 464 & 297 \\
1923 & 995 & 372 & 692 & 1152 & 516 & 258 \\
1924 & 395 & 347 & 499 & 1111 & 437 & 224 \\
1925 & 286 & 409 & 417 & 1166 & 458 & 272 \\
1926 & 318 & 350 & 374 & 1653 & 474 & 217 \\
1927 & 311 & 316 & 342 & $512 *$ & 447 & 184 \\
1928 & 303 & 275 & 394 & 2082 & 508 & 263 \\
1929 & nd & nd & nd & nd & nd & nd \\
1930 & 630 & 654 & 850 & 1694 & 1150 & 636 \\
1931 & 739 & 872 & 1206 & 1532 & 1126 & 666 \\
1932 & 771 & 809 & 1115 & 2291 & 1047 & 749 \\
1933 & 995 & 788 & 1352 & 1936 & 751 & 524 \\
1934 & 402 & 320 & 1140 & 1567 & 957 & 658 \\
1935 & 794 & 729 & 1154 & 1614 & 1067 & 648 \\
1936 & 756 & 886 & 1103 & 1422 & 973 & 623 \\
\hline
\end{tabular}

* À noter qu'en 1927, une épidémie de typhoïde, qui a mobilisé les énergies du personnel médical du Service de santé, frappait Montréal, ce qui peut expliquer le faible nombre des inscriptions estivales pour cette année-là.

Sources: Montréal, Bulletin d'hygiène, volumes 6 à 22 (1920-1936).

23. Ceci en supposant que tous les enfants fréquentaient la Goutte de lait durant toute leur première année et que les enfants qui prolongeaient leur fréquentation au-delà de cet âge compensaient pour ceux qui abandonnaient avant d'atteindre l'âge d'un an. Si on prend pour acquis qu'ils étaient assidus à la clinique durant seulement six mois, on obtient un taux de fréquentation de 45,6\% (14 294/(0,5 x $20622+21044)$.

24. Il est difficile d'évaluer quelle proportion de la population montréalaise, et donc des naissances, était couverte par les cliniques à chacun des stages de leur développement, d'autant 
Comme on peut le voir, les inscriptions faisaient un bond important durant les mois de juillet et août, la période la plus propice aux diarrhées infantiles. Il faut dire que, durant les années 1920, cinq des cliniques municipales qui desservaient les populations dispersées sur les territoires les plus éloignés du centre ne fonctionnaient que durant cette saison, ce qui contribuait certainement à hausser le chiffre des inscriptions estivales. Ce phénomène ne peut cependant expliquer à lui seul les très grands écarts enregistrés. En fait, à la lumière du tableau 2 , on peut certainement dire que les mères étaient plus enclines à se déplacer durant les grandes chaleurs, soit lorsque les risques de mortalité pour leurs enfants étaient les plus élevés. Mentionnons que durant l'été, des concours d'assiduité, sur lesquels nous reviendrons plus loin, étaient organisés afin justement de les inciter à faire examiner leur enfant régulièrement. Enfin, le tableau montre aussi que les écarts entre les inscriptions estivales et les autres mois de l'année diminuent après 1929, alors que la Ville étend son réseau et qu'elle consentira de nouveaux efforts pour rejoindre individuellement les mères, comme on le verra dans la deuxième partie de ce texte.

Durant les vingt premières années de fonctionnement des Gouttes de lait, on peut donc dire que les promoteurs sont parvenus à convaincre un nombre croissant de femmes de s'y rendre. Ainsi, en 1922, dans son rapport annuel, le docteur Boucher, directeur du Service de santé de la Ville de Montréal, constatait que les consultations étaient «bien fréquentées» et que, en général, les bébés inscrits se présentaient «assez régulièrement pour qu'il soit possible de suivre leur développement d'une manière satisfaisante ${ }^{25}$ ». Selon les données recueillies, il estimait à $43 \%$ la proportion des enfants amenés au moins une fois à la Goutte de lait durant leur première année, un chiffre jugé encourageant, car, selon lui, «il [était] difficile d'espérer augmenter cette proportion à plus de 50 pour cent $^{26}{ }^{\prime}$. Le même rapport déplorait cependant que seulement $34 \%$ des bébés aient été inscrits au cours des trois premiers mois suivant leur naissance, le moment le plus crucial pour la survie des nourrissons, et il concluait que «les résultats [quant à la lutte contre la mortalité infantile] seraient encore meilleurs si la

plus que des mères provenant de l'extérieur des paroisses où se retrouvaient les Gouttes de lait pouvaient aussi les fréquenter. Par ailleurs, on peut dire qu'environ $20 \%$ des ménages montréalais avaient sans doute des ressources suffisantes pour avoir recours à un médecin et n'étaient donc pas visés par les Gouttes de lait (Terry Copp, The Anatomy of Poverty (Toronto, McClelland and Stewart, 1974), 36).

25. RASS (1922), 38.

26. Ibid., 83. Ce taux de fréquentation est certainement gonflé, étant donné qu'en 1922, on comptabilisait toujours plusieurs fois les enfants lorsqu'ils s'absentaient de la clinique durant de trop longues périodes. 


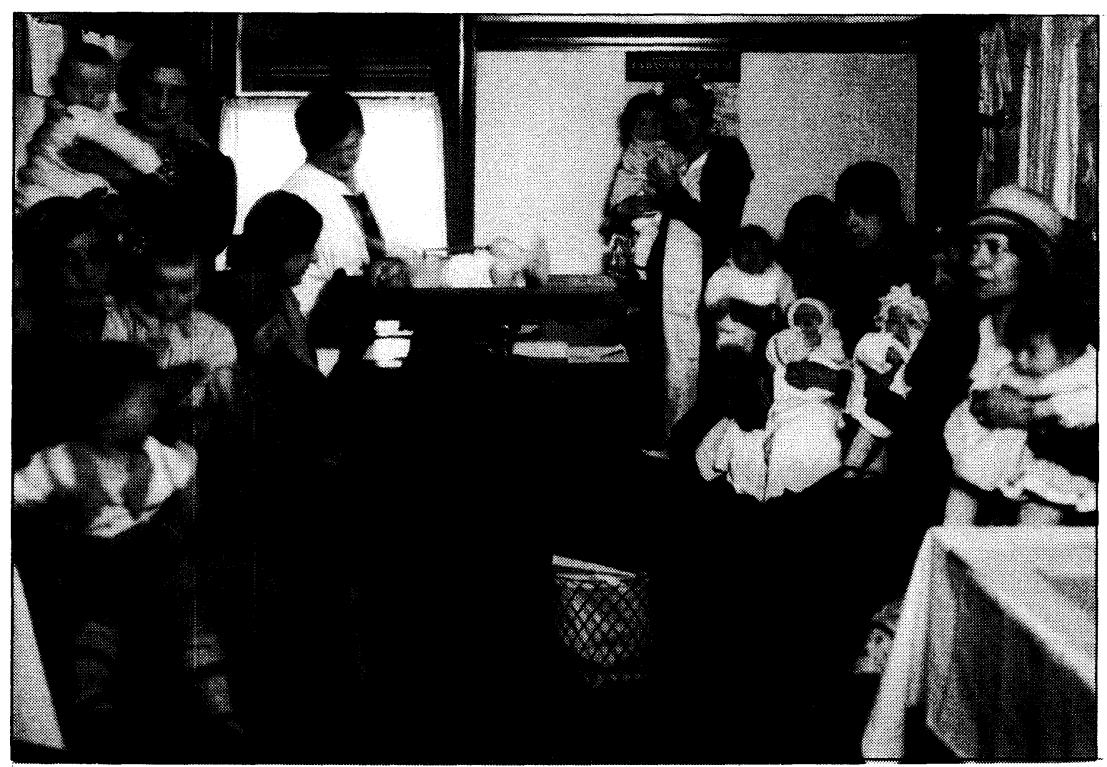

Une clinique du Service de santé en 1932. Ville de Montréal, Gestion de documents et archives. Z96-4.

fréquentation était plus assidue et surtout si l'on n'attendait pas que l'enfant soit malade pour le conduire à la consultation ${ }^{27}$ ».

Ainsi, l'irrégularité des fréquentations, un problème souvent mentionné jusqu'au milieu des années 1920, semblait tenir au fait que, à cette époque, bien des femmes ne voyaient pas la nécessité de se déplacer lorsque leur enfant se portait bien. C'est donc dire que le message des promoteurs au sujet de l'importance des conseils dispensés à la Goutte de lait pour prévenir les problèmes de santé et la mortalité a mis un certain temps à porter fruit et ce, pour différentes raisons. En premier lieu, il faut souligner que certains propagandistes des Gouttes de lait ont parfois tenu un discours qui ne s'accordait pas tout à fait avec celui du Service de santé de la Ville, en insistant par exemple pour que les mères y amènent leurs enfants surtout lorsqu'ils étaient malades: «[...] les mamans seraient désormais inexcusables de ne pas présenter à la Goutte de lait leur bébé malade (le souligné est de nous), sous prétexte de dérangement, de défiance ridicule ou d'une certaine fierté28.» Comme cet observateur le laisse sous-entendre, les femmes pouvaient avoir bien des motifs pour ne pas avoir recours à

27. Idem.

28. «La Goutte de lait», Bulletin paroissial Immaculée-Conception, 9,1 (novembre 1917): 5 . 
ce service: crainte de se voir assimiler aux classes les plus pauvres, méfiance envers le personnel médical et bénévole qui prétendait leur apprendre comment nourrir et soigner leur enfant, mais aussi refus ou impossibilité de se déplacer.

Des enquêtes à domicile effectuées par les «dames zélatrices» des comités paroissiaux affiliés à la Commission centrale de la Goutte de lait - une structure mise en place par la FNSJB en 1914 - démontrent qu'effectivement il pouvait être très difficile pour les mères de se rendre à la Goutte de lait lorsqu'elles étaient très pauvres ou chargées d'enfants. En janvier 1916, par exemple, la responsable du comité de la paroisse Saint-Anselme rapportait: «Depuis le premier janvier, la moyenne de la fréquentation est de 10 par jour. Il a fallu donner des vêtements pour faciliter [sic] les mères et leur bébé à venir à la Goutte de lait ${ }^{29}$.» Le manque de vêtements pour les mères et les enfants, mais aussi la difficulté de trouver une gardienne reviennent assez souvent dans les rapports de ces comités pour en conclure qu'il s'agissait d'une situation très répandue: «Bien des mères ne se rendent pas à la Goutte de lait dans certains cas faute de gardienne pour prendre soin de plusieurs enfants; la grande pauvreté en prive un certain nombre d'autres et il est difficile de convaincre une autre catégorie de mères qui pourraient facilement fréquenter la Goutte de lait ${ }^{30} . »$

Ces rapports en provenance de bénévoles féminines permettent donc de constater que l'extrême pauvreté dans laquelle vivaient certaines mères de famille n'était guère propice à la fréquentation des cliniques, alors même que ce service leur était plus particulièrement destiné. Ils révèlent également que le support mutuel de gardiennage que les femmes pouvaient s'offrir comportait certaines limites. À une époque où beaucoup de familles étaient encore relativement nombreuses, il pouvait en effet être difficile de confier ses enfants à une autre femme ${ }^{31}$. Même si dans les quartiers populaires il était courant

29. Procès-verbal de l'assemblée de la Commission centrale des Gouttes de lait (PVCC), 13 janvier 1916, Archives nationales du Québec, dépôt de Montréal (ANQM), Fonds de la Fédération nationale Saint-Jean-Baptiste (FNSJB).

30. PVCC, 9 septembre 1915, Rapport du comité Hochelaga, ANQM, Fonds FNSJB. Ce commentaire laisse également voir que certaines femmes opposaient une forme de résistance. Voir aussi PVCC, 9 septembre 1915, Rapport du comité Saint-Pierre, ANQM, Fonds FNSJB.

31. En 1915, le taux de natalité pour Montréal était de 36,96 pour mille. Ce n'est qu'à partir de 1923 qu'il est descendu définitivement en dessous des 30 pour mille, se situant cette année-là à 27,06 pour mille (Québec, Annuaire statistique (1917), 79 et (1925), 110). Par ailleurs, selon les tableaux présentés par Jacques Henripin dans La population du Québec d'hier à demain, les femmes nées entre 1896 et 1901, et qui ont donc commencé à donner naissance à partir de 1915 environ, ont eu une descendance finale de 3,4 enfants en moyenne (Jacques Henripin, «Tableaux sur l'évolution séculaire de la fécondité», dans Jacques Henripin et Yves Martin, dir., La population du Québec d'hier à demain (Montréal, Presses de l'Université de Montréal, 1991), 48. 
de vivre près de sa parenté, solliciter une aide de cette nature, surtout sur une base régulière, ne semblait pas aller de soi. En fait, pour favoriser de manière optimale la fréquentation, il aurait sans doute fallu ouvrir les cliniques le soir, alors que les pères étaient disponibles pour prendre la relève auprès des autres enfants. C'est du moins ce que laissent entendre les commentaires de certaines déléguées à la Commission centrale. Mais les dirigeantes de la FNSJB n'admettaient pas cette idée; à leur avis, le soir, la place des femmes était au foyer ${ }^{32}$.

De nombreux obstacles empêchaient donc les femmes les plus démunies et les mères de familles nombreuses de fréquenter les cliniques, surtout sur une base régulière. Dans ce contexte, il n'est donc pas étonnant d'apprendre que, au cours des années 1910 en particulier, une bonne proportion de celles qui s'y présentaient le faisaient pour des raisons très pragmatiques, soit que leur bébé nécessitait des soins urgents, soit que leur pauvreté les poussait à venir chercher du lait. La distribution gratuite de lait semblait en effet particulièrement attirer la clientèle, au point où les promoteurs ont senti à plusieurs reprises la nécessité de rappeler aux mères qu'elles étaient invitées à se présenter aux consultations «non seulement pour recevoir du lait mais pour faire examiner leur enfant ${ }^{33} »$. Pour tenter de corriger la situation, un comité paroissial adopta même une résolution à l'effet «que le lait ne soit donné que si les mères se présentent à la clinique avec leur bébé ${ }^{34}{ }^{\prime}$, ce qui signifie que beaucoup de femmes ne se donnaient même pas la peine de les y amener. En fait, tout porte à croire que pour les mères les plus pauvres, la Goutte de lait représentait davantage un lieu où elles pouvaient obtenir gratuitement une denrée essentielle au bien-être de leur famille qu'un endroit où aller chercher des conseils. D'ailleurs, le lait qui en principe devait servir à alimenter le nouveau-né pouvait tout aussi bien être consommé par les autres enfants, comme le rapportaient les dames du comité de la paroisse Saint-Henri ${ }^{35}$.

32. «Quelques dames font remarquer qu'il était toujours difficile pour les mères de laisser l'après-midi parce qu'elles n'ont pas de gardienne et que souvent lorsque les enfants sont nombreux, ils ne sont pas tous également habillés pour sortir. Auclair fait remarquer que GérinLajoie avait déjà demandé d'essayer d'habituer les femmes à sortir le jour pour leur propre santé d'abord, et pour qu'elles soient à leur foyer le soir. Plusieurs déléguées font remarquer que la plupart préfêrent sortir le soir, lorsque le mari est à la maison elles sont alors sans inquiétude» (PVCC, 9 décembre 1916, ANQM, Fonds FNSJB).

33. Discours d'ouverture des Gouttes de lait de mademoiselle Auclair, 1914, ANQM, Fonds FNSJB. Dans le même discours Maria Auclair affirmait: «J'ai rencontré moi-même des mères qui ne voulaient que se procurer du lait, tandis que le médecin constatait que l'enfant était si malade qu'il aurait d'abord besoin d'un traitement.»

34. "Chroniques des œuvres», La bonne parole, 11,12 (février 1915): 4. Ce fait révèle également qu'un certain laxisme existait au niveau du fonctionnement des Gouttes de lait, car, en principe, le lait ne devait être distribué qu'après une évaluation médicale du cas.

35. PVCC, 17 décembre 1914, ANQM, Fonds FNSJB. 
Les pratiques en usage dans certaines consultations paroissiales, au tout début surtout, contribuaient d'ailleurs à entretenir une certaine confusion au sujet des objectifs de l'œuvre. Les rapports des comités affiliés à la Commission centrale de la FNSJB révèlent, en effet, qu'il arrivait très souvent que les médecins ne se présentaient pas pour recevoir les femmes en consultation, ce qui était de nature à convaincre les mères que les Gouttes de lait visaient essentiellement à distribuer du lait ${ }^{36}$. Cette conception était assez largement répandue pour qu'en 1915, le docteur Boucher demande aux dames bénévoles d' «arracher aux mères cette idée [que les Gouttes de lait n'étaient là que pour distribuer du lait $]^{37}{ }_{\Perp}$. En 1917, dans son rapport annuel, il constatait par contre une certaine amélioration puisqu'il écrivait: «Nous avons réussi à bien faire comprendre à tous les comités qu'ils doivent partout viser à faire de l'éducation ${ }^{38}$.»

Les comités semblaient, en effet, avoir mieux compris leur rôle puisque pour attirer davantage de femmes dans les cliniques et pouvoir faire leur éducation sur une base régulière, ils avaient mis au point un moyen qui allait s'avérer très efficace: l'organisation de concours d'assiduité. Orchestrés durant la première année par le Bureau central des Gouttes de lait, puis par la Ville, ces concours visaient à «augmenter l'intérêt que portent les mères de familles aux Gouttes de lait» et surtout à les convaincre de faire «des visites hebdomadaires et de ne pas attendre que les enfants soient malades pour leur faire profiter des avantages des Gouttes de lait ${ }^{39}{ }$. Chaque Goutte de lait paroissiale était chargée de l'organisation d'un concours local, les bébés «les plus méritants» pouvant prendre part au concours général. Les conditions de participation étaient simples; il suffisait que la mère se présente une quinzaine de semaines consécutives durant l'été pour que l'enfant soit pesé et examiné par le médecin. Cinq prix en argent, variant entre $1 \$$ et $10 \$$, en plus d'articles de lingerie pour enfant, étaient décernés par la paroisse et une vingtaine, variant entre $1 \$$ et $15 \$$, au concours généra ${ }^{40}$.

Le nombre et surtout la générosité des prix offerts étaient certainement de nature à mousser la popularité des Gouttes de lait. Ces sommes d'argent étaient importantes pour l'époque, les premiers prix

36. Voir par exemple «Chroniques des œuvres», La bonne parole, 11,12 (février 1915): 4. Cet absentéisme témoigne selon nous de la position conflictuelle dans laquelle devaient se retrouver certains médecins; entre les exigences de leur pratique privée et leur jour de garde à la consultation, certains d'entre eux ont eu vite fait de choisir.

37. PVCC, 8 avril 1915, ANQM, Fonds FNSJB.

38. $\operatorname{RASS}(1917): 28$

39. La bonne parole, 4,6 (août 1916): 2.

40. «Les Gouttes de lait», Bulletin paroissial Immaculée-Conception, 8,8 (juin 1917): 3. 
pouvant même équivaloir au salaire hebdomadaire d'un ouvrier. Pour bien des mères de famille, la possibilité de gagner n'était sûrement pas à dédaigner. En fait, il semble bien que ces concours aient joué un rôle important dans l'augmentation du nombre des inscriptions et de la fréquence des visites puisqu'en 1923, dans son rapport annuel, le directeur du Service de santé, après avoir constaté une baisse de la fréquentation des cliniques municipales, commentait: «D'après les renseignements qui m'ont été fournis, la principale cause de ce ralentissement a été la suppression du concours annuel depuis 2 ans. [...] J'espère qu'il sera possible de rétablir ces concours officiels qui sont un puissant stimulant à l'assiduité aux consultations durant l'été ${ }^{41}$.»

C'est donc en partie par le biais de concours que les promoteurs des Gouttes de lait sont parvenus à s'attirer une clientèle plus considérable et surtout plus régulière, en particulier l'été. Plus que la promesse de recevoir des conseils utiles - et même, selon les médecins, essentiels à la survie de leurs nouveau-nés -, cette stratégie a suscité l'enthousiasme de bien des mères. En fait, les rapports annuels de la Ville et ceux des déléguées paroissiales auprès de la Commission centrale incitent à conclure que, jusqu'en 1920 environ, l'aide matérielle que pouvaient offrir ces cliniques, que ce soit du lait ou des prix, ou encore la possibilité de faire examiner gratuitement un enfant malade, comptaient davantage pour les mères que la perspective d'une consultation axée uniquement sur l'éducation et visant à leur faire adopter des pratiques inapplicables ou qui allaient à l'encontre de leurs habitudes. À ce chapitre, si on ne peut connaître l'attitude de ces femmes en regard de tous les conseils dispensés à la Goutte de lait, on peut tout de même souligner que la promotion de l'allaitement maternel a continué de rencontrer une forte résistance ${ }^{42}$.

Par contre, les progrès enregistrés tout au long de ces deux décennies, que l'on considère le nombre de bébés inscrits ou l'assiduité, ont eu certainement pour résultat d'exposer davantage de femmes au message hygiéniste. De concert avec la pasteurisation croissante du lait et l'introduction de certains vaccins, la diffusion des mesures élémentaires

41. RASS (1923), 97. Le tableau 1 fait état de cette baisse; le chiffre des inscriptions pour les consultations municipales passant de plus de 4517 en 1922 à 4005 en 1923. En 1924, le nombre total des inscriptions est descendu à 8600 , ce qui provient surtout des changements introduits dans la manière de les comptabiliser.

42. Selon les statistiques en provenance de la paroisse Immaculée-Conception, par exemple, seulement le tiers des femmes qui fréquentaient la clinique allaitaient leurs enfants en 1928, et encore, ce chiffre comprenait celles qui utilisaient une alimentation mixte («La Goutte de lait», Bulletin paroissial Immaculée-Conception, 19,4 (février 1928): 18). Autre indice du faible taux d'allaitement, en 1931, la Ville entreprit une nouvelle campagne de sensibilisation au moyen d'affiches exposées dans les Gouttes de lait et envoyées par la poste aux nouvelles mères (RASS (1931), 124-125). 
en matière d'hygiène s'est traduite par une diminution constante de la mortalité infantile au cours des années $1920^{43}$. Au début des années 1930, elle était cependant encore élevée et de nouveaux efforts vont être mis en œuvre par la Ville de Montréal pour amener toujours davantage de mères aux consultations. Les témoignages entendus démontrent que les femmes s'y rendaient plus volontiers, mais pas toujours pour des raisons exclusivement médicales. En outre, ils révèlent que certaines conditions devaient être réunies pour favoriser cette fréquentation.

\section{FRÉQUENTER LES GOUTTES DE LAIT ENTRE 1930 ET 1965}

$\mathrm{Au}$ cours de la période qui s'amorce avec les années 1930, les Gouttes de lait sont devenues une véritable institution. Elles étaient d'abord plus accessibles, puisque la Ville inaugura 20 nouvelles consultations en 1929 seulement et continua d'élargir ce réseau par la suite, de manière à atteindre la plupart des quartiers montréalais ${ }^{44}$. En fait, durant cette seconde phase d'expansion, toutes les mères, et non plus seulement celles qui habitaient les secteurs les plus pauvres, étaient invitées à se prévaloir de ce service. Les Gouttes de lait bénéficiaient également d'une publicité plus large. À partir de 1932, le Service de santé s'efforça de rejoindre plus systématiquement les nouvelles mères, immédiatement après leur accouchement, au moyen de visites à domicile effectuées par des infirmières. Ces dernières devaient rappeler aux mères l'existence des cliniques et les inciter à $s^{\prime} y$ rendre $^{45}$. Si une mère s'absentait de la consultation durant plu-

43. Selon le bulletin d'hygiène, le taux de mortalité infantile à Montréal a décrû parallèlement à l'augmentation du pourcentage de vaches tuberculinées et de lait pasteurisé. Ainsi, entre 1915 et 1919 , alors que $1,9 \%$ des vaches étaient tuberculinées et que $44,88 \%$ du lait était pasteurisé, le taux de mortalité infantile se situait, en moyenne pour les cinq années, à 183,03 pour mille; pour la période $1925-1929$, ces proportions étaient respectivement de $70,5 \%$, $85,98 \%$ et de 126,8 pour mille; en $1935-1937$, ils avaient atteint $100 \%, 94,39 \%$ et 77,2 pour mille («Le lait et la mortalité à Montréal», Bulletin d'hygiène de la Ville de Montréal, 25,5 (septembre-octobre 1939): 9).

44. En 1930 , le nombre de cliniques municipales était de 36 pour un total de 68 en tenant compte des consultations privées anglophones et francophones. En 1935, il existait 42 consultations municipales et 31 privées pour un total de 73. En 1940, le nombre des consultations municipales était de 47 et celui des consultations privées de 28 , donc 75 au total et, enfin, en 1950, la Ville contrôlait 61 des 87 consultations existantes. Par la suite, leur nombre s'est stabilisé et a même décrû avec l'absorption, par le Service de santé, des cliniques de la Fédération d'hygiène infantile (RASS $(1930,1935,1940,1950)$.

45. RASS (1932), 38. Les visites à domicile existaient depuis le début de la création des cliniques, comme en témoignent celles qui étaient effectuées par les dames zélatrices de la FNSJB. Assez rapidement, du moins dans les cliniques municipales, ce sont des infirmières ou des aide-infirmières qui furent chargées de cette tâche, mais ce n'est que durant les années trente que la Ville parvint à mettre au point un système qui lui permettait de leur transmettre rapidement la liste des femmes qui venaient de donner naissance dans chacune des paroisses (RASS (1932), 38-39). 
sieurs semaines, l'infirmière devait lui faire une visite de rappel pour en connaître la raison et la convaincre d'y revenir ${ }^{46}$. À ces visites à domicile, venaient s'ajouter une série de brochures, de dépliants et d'affiches que la Ville leur faisait parvenir dès la naissance de l'enfant ${ }^{47}$. Sollicitées de la sorte, il semble que plus de femmes ont répondu à l'appel, du moins si on se fie à l'absence de doléances à ce chapitre dans les rapports annuels du Service de santé. Le tableau suivant montre la progression du nombre des enfants inscrits entre 1935 et 1960 par rapport aux naissances pour Montréal.

TABLEAU 3

Inscriptions aux Gouttes de lait privées et aux consultations municipales, nombre annuel des naissances pour Montréal et taux de fréquentation estimé

$1935-1960$

\begin{tabular}{|c|c|c|c|c|}
\hline Années & $\begin{array}{l}\text { Nombre de } \\
\text { cliniques }\end{array}$ & $\begin{array}{c}\text { Nombre } \\
\text { d'inscriptions }\end{array}$ & $\begin{array}{l}\text { Nombre de } \\
\text { naissances }\end{array}$ & $\begin{array}{l}\text { Taux de } \\
\text { fréquentation } \\
\text { estimé }\end{array}$ \\
\hline 19.35 & 72 & 19481 & 17786 & $53.8 \cdot 72.1 \%$ \\
\hline 1940 & 75 & 17405 & 19448 & $47.6-01.1 \%$ \\
\hline 1945 & 8.3 & 20168 & 22775 & $42,9-57,6 \%$ \\
\hline 1950 & 87 & 24667 & $25 \quad 177$ & $49.6-65.9 \%$ \\
\hline 1955 & 70 & 30734 & 28510 & $53.9-71.9 \%$ \\
\hline 1960 & 6.5 & 41181 & 28409 & $70.9-45.2 \%$ \\
\hline
\end{tabular}

Sources: RASS, 1935, 1940, 1945, 1950, 1953-1954-1955 et 1959-1960; Québec, Annuaire statistique, 19.

En reprenant le même mode de calcul que précédemment ${ }^{48}$, on obtient des taux de fréquentation variant de $53,8 \%$ à $72,1 \%$ pour l'année 1935 et de $70,9 \%$ à $95,2 \%$ pour 1960 . Le tableau montre qu'il y a eu un déclin de la fréquentation durant la guerre, ce qui peut être attribuable à la présence plus nombreuse des femmes mariées sur le marché du travail, avant qu'elle ne remonte dans les années 1950 pour atteindre de très fortes proportions au début des années 1960. Mentionnons également que, selon des données provenant des Gouttes de lait privées, $48 \%$ des bébés nés dans l'ensemble des paroisses qu'elle desservait fréquentaient ces cliniques en 1948. Dans certains secteurs particulièrement pauvres, les taux de fréquentation stagnaient en-

46. $\mathrm{D}^{\mathrm{r}} \mathrm{A}$. Groulx, «Le rôle de l'infirmière visiteuse du service de santé», Bulletin d'hygiène de la Ville de Montréal, 26,4 (juillet-août 1940): 2-12.

47. RASS (1932), 39.

48. C'est-à-dire en tenant compte des naissances pour chacune des années précédentes. En 1934, elles se chiffraient à 18463 ; en 1939 à 17661 ; en 1944 à 24 193; en 1949 à 24489 ; en 1954 à 28420 et en 1959 à 29640 . 
dessous des $40 \%$, mais ailleurs ils pouvaient atteindre les 60 et même les $90 \%{ }^{49}$. Si, dans leur ensemble, les Gouttes de lait étaient plus fréquentées durant cette période, c'est donc non seulement parce qu'on leur a fait davantage de publicité, mais aussi parce qu'elles rejoignaient des femmes qui bénéficiaient d'un niveau de vie plus élevé.

Par rapport à 1910, la mortalité infantile avait beaucoup diminué au début des années 1930, se situant alors à 124,8 pour mille. En 1939, elle était de 71,5 pour mille, un taux encore élevé par rapport aux autres provinces canadiennes ${ }^{50}$, mais qui, selon le Service de santé, était dû en bonne partie à la surmortalité des enfants «illégitimes». En effet, le taux de décès chez ces derniers était particulièrement désastreux $(288,2$ pour mille), alors que chez les bébés «légitimes», il était de 57,1 pour mille ${ }^{51}$. Cette année-là, le rapport annuel du Service de santé faisait aussi remarquer que, depuis 1934, les «diarrhées-entérites», qui avaient longtemps provoqué la majorité des décès chez les nouveau-nés, étaient à l'origine de moins d'un décès sur cinq, alors que plus de 40 pour cent étaient dus désormais à des causes prénatales (naissances prématurées, débilité congénitale, malformations) ${ }^{52}$.

Du seul point de vue de la santé publique, on peut donc dire que la majorité des femmes qui ont été interviewées, et dont les témoignages constituent la source principale sur laquelle se fonde cette deuxième section, ont vécu leurs grossesses dans un contexte bien différent. Plusieurs d'entre elles ont aussi fréquenté les Gouttes de lait à partir de années 1940, soit durant une époque de relative prospérité

49. Ainsi, les Gouttes de lait de deux paroisses situées à Verdun (Notre-Dame Auxiliatrice et Notre-Dame de la paix), de même que celles des paroisses ImmaculéeConception, Nativité, Sacré-Cœur, Sainte-Cunégonde, Saint-Euzèbe, Saint-Jean-Baptiste, SaintJoseph, Saint-Stanislas et celle de Pointe-aux-Trembles, avaient un taux de fréquentation variant entre 29,6 et 45,1\%. Dans les paroisses Notre-Dame de Lourdes, Notre-Dame des Sept-Douleurs (Verdun), Sainte-Jeanne d'Arc, Saint-Alphonse, Sainte-Brigide, Sainte-Catherine, Sainte-Cécile, Saint-Enfant-Jésus, Saint-Pierre et Saint-Vincent-de-Paul, les taux se situaient entre 48 et $56,4 \%$. Dans les paroisses Notre-Dame du Sacré-Cœur et Saint-Nazaire (toutes deux situées à Ville de LaSalle), Notre-Dame du Perpétuel Secours, Sainte-Élizabeth, Sainte-Gertrude, Saint-Pierre aux Liens et Saint-Vital, les taux étaient de 61,5 à 94,7\% (Les Gouttes de lait paroissiales, Rapport annuel pour l'année 1948, Archives de la Ville de Montréal, $3^{e}$ série, dossier 92.210.3).

50. Pour l'ensemble du Canada, le taux de mortalité infantile à cette date était de 61 pour mille. En Ontario il était de 46 pour mille (Canada, Ministère de l'Industrie et du Commerce, Annuaire statistique du Canada (1942), 97).

51. RASS (1939), 51. Au Québec, le taux de mortalité infantile, naissances légitimes et illégitimes confondues, continuera de diminuer par la suite: entre 1940 et 1944, il s'est maintenu en moyenne à 64,5 pour mille; entre 1945 et 1949 à 54,3 pour mille; entre 1950 et 1954 à 40,9 pour mille; entre 1955 et 1959 à 29,9 pour mille et entre 1960 et 1964 à 22,2 pour mille (RASS (1968-1969), 21).

52. RASS (1939), 52. 
économique, alors que la hausse des salaires améliorait de façon notable les condicions de vie des familles d'ouvriers et de petits salariés. Enfin, ces années ont aussi été témoin d'une baisse des taux de natalité et de la fécondité, comparativement à la période précédente ${ }^{53}$.

L'échantillon constitué au moyen des entrevues comprend 31 femmes qui ont toutes fréquenté les Gouttes de lait, avec un ou plusieurs de leurs enfants. Il est à noter que le fait d'avoir eu recours à ces cliniques représentait le seul critère de sélection, ce qui, bien sûr, éliminait celles qui, pour une raison ou une autre, ont refusé d'utiliser ce service ${ }^{54}$. Pour la plus grande part, soit dans 26 cas sur 31 , ces femmes ont vécu leur première grossesse entre 1935 et $1949^{55}$. En moyenne, elles ont élevé 4,4 enfants, en comptant ceux qui ont été adoptés (au nombre de 4). La répartition selon la taille de la famille est présentée dans le tableau suivant.

TABLEAU 4

Répartition du nombre d'enfants par femme

\begin{tabular}{|c|c|}
\hline $\begin{array}{c}\text { Nombre } \\
\text { d'enfants }\end{array}$ & $\begin{array}{c}\text { Nombre de } \\
\text { femmes }\end{array}$ \\
\hline $1-2$ & 8 \\
$3-5$ & 15 \\
$6-9$ & 6 \\
10 et plus & 2 \\
Total & 31 \\
\hline
\end{tabular}

53. Les taux de natalité pour Montréal se situaient aux environs de 28 pour mille durant les années vingt; au cours de la crise, ils sont descendus jusqu'à 19 pour mille, pour ensuite remonter jusqu'à 26 pour mille durant le «Baby Boom» des années quarante et cinquante (Québec, Annuaire statistique, 1920-1955). Par ailleurs, la descendance finale des femmes nées entre 1911 et 1916, pour Montréal, s'établit à 2,6 enfants et, pour celles nées entre 1916 et 1921 , à 2,8 (Henripin, «Tableaux sur l'évolution», 48).

54. Toutes ces femmes, sauf deux, ont été sollicitées au moyen d'un communiqué publié dans un réseau d'hebdomadaires locaux (le Super Hebdo), couvrant plusieurs quartiers de Montréal. Une seule s'est mariée durant les années vingt, en 1925 plus précisément, et une a convolé en 1956; parmi les 29 autres, 12 se sont mariées durant les années trente, dont neuf entre 1935 et 1939, et 17 durant les années quarante, à une exception près, avant 1946.

55. Onze d'entre elles avaient déjà complété leur famille à la fin des années quarante, 14 à la fin des années cinquante, tandis que 6 autres ont eu leur dernier enfant dans les années soixante, la dernière naissance survenant en 1966. 
Comme on peut s'en rendre compte, les trois quarts de ces femmes ont donné naissance ou ont élevé cinq enfants ou moins, et seulement deux en ont élevé plus de dix, dont l'une, onze et l'autre, quinze. Douze d'entre elles toutefois ont vécu des fausses-couches ou ont perdu un enfant à la naissance ou en bas âge, ce qui signifie que le nombre moyen de grossesses est plus élevé, se situant à cinq.

Un peu moins de la moitié de ces femmes, soit 14 sur 32, étaient mariées à des travailleurs manuels, dont cinq à des ouvriers qualifiés ${ }^{56}$, 16 à des cols blancs, travailleurs de bureaux et des services (transport et vente) et la dernière à un professionnel (pharmacien). La présence des femmes des deux derniers groupes au sein de l'échantillon atteste que les Gouttes de lait visaient désormais une clientèle plus large. Enfin, ajoutons qu'elles ont élevé leur famille dans 24 paroisses différentes de la Ville de Montréal et de Verdun, où il existait quelques Gouttes de lait privées, et qu'elles ont fréquenté 29 consultations différentes, dont environ la moitié appartenaient au réseau de la Ville de Montréal ${ }^{57}$.

Quand on leur a demandé comment elles avaient appris l'existence des Gouttes de lait, plusieurs d'entre elles ont simplement répondu que «c'était dans la paroisse», que «tout le monde le savait», que «tout le monde en parlait» ou encore que «tout le monde y allait». Près du tiers ont également affirmé que leur mère les avaient fréquentées avant elles, signifiant par là qu'elles ne faisaient en quelque sorte que poursuivre la tradition («c'était l'esprit de famille», affirmait une informatrice). C'est donc dire que, parmi la première génération de femmes qui a fréquenté ces cliniques, plusieurs étaient suffisamment satisfaites pour transmettre à leur fille une image positive de ce service. Deux ont aussi indiqué que les prêtres de la paroisse en faisaient la promotion en chaire, quelques-unes ont dit avoir été conseillées par une sœur, une voisine ou une infirmière ${ }^{58}$ et cinq seulement ont affirmé que c'était à l'instigation de leur médecin accoucheur. Cette faible proportion est un peu surprenante, car on aurait pu croire que les praticiens étaient les mieux placés pour inciter les femmes à s'y rendre. Certains médecins n'étaient peut-être pas si

56. Machiniste, lamineur, tourneur, électro-typeur, lithographe.

57. Les paroisses les plus souvent mentionnées sont: Très-Saint-Rédempteur (3); SaintPierre Claver (3); Saint-Ambroise (4); Saint-Jean-Berchmans (4). Les Gouttes de lait SaintArsène; Immaculée-Conception, École Baril, Saint-Ambroise, Saint-Jean-Berchmans et SacréCœur ont été mentionnées plus d'une fois.

58. Dans certains cas, il s'agissait d'une infirmière de la Ville de Montréal, mais certaines ont aussi précisé qu'il s'agissait d'une infirmière de la compagnie d'assurance-vie La Métropolitaine qui leur avait dispensé des soins postnatals ou bien d'une infirmière de l'hôpital où elles avaient accouché. 
empressés d'en faire la promotion ${ }^{59}$ ou bien les femmes avaient oublié ce détail, entre autres raisons parce qu'elles connaissaient l'existence des Gouttes de lait avant même qu'ils ne leur en parlent.

À la lumière de ces témoignages, on peut donc dire que les Gouttes de lait étaient bien ancrées dans la communauté paroissiale et qu'elles étaient parvenues à se tailler une place non négligeable dans la vie de bien des femmes. Malgré une assiduité assez variable, la plupart des femmes interviewées considéraient qu'elles ne pouvaient complètement ignorer ce service et un peu moins des deux tiers des femmes de l'échantillon (19 sur 31) y ont amené tous leurs enfants: «Le bébé, c'était primordial. Ça, c'était... c'était sacré, ça. Tu ne pouvais pas arriver et rater $\mathrm{ça}^{60} . »(\mathrm{E} 28)$ Une informatrice allait même jusqu'à dire que «c'était compris ça dans l'élevage d'une famille que la Goutte de lait prenait part... c'est le rituel. C'était écrit ça.» (E19)

Celles qui ont eu deux ou trois enfants étaient les plus susceptibles d'avoir fréquenté les Gouttes de lait régulièrement, et avec chacun d'eux, tandis que celles qui ont continué de s'y rendre même après le quatrième ou le cinquième enfant ont mentionné qu'une femme de la parenté (mère, belle-mère ou une sœur) pouvait garder les plus vieux à l'occasion, ou bien que leurs enfants étaient suffisamment espacés pour que les plus âgés fréquentent l'école au moment où elles devaient s'y présenter avec les plus jeunes. Une des informatrices faisait d'ailleurs remarquer que sa belle-sœur, qui avait neuf enfants, ne fréquentait pas ces cliniques et une autre affirmait qu'il était rare de rencontrer à la Goutte de lait des femmes accompagnées de plus de deux enfants. Leurs témoignages à ce chapitre démontrent qu'un nombre moins élevé d'enfants et le support de l'entourage facilitaient grandement la fréquentation. La baisse de la fécondité, amorcée durant les années 1930 en raison de la crise et qui s'est maintenue au cours des années 1940 et 1950 , et le recours plus fréquent à la contraception, qui permettait d'espacer les naissances ${ }^{61}$, se sont donc avérées des conditions préalables importantes pour assurer le plus grand succès des Gouttes de lait.

59. Au cours des années trente, les revues médicales publient encore des textes provenant de médecins qui s'insurgent contre les Gouttes de lait parce que les infirmières y prennent de trop grandes libertés professionnelles. Voir «La garde m'a dit», L'Action médicale (octobre 1939): 191.

60. Entrevue $\mathrm{n}^{\circ} 28$; à l'avenir, chacun des extraits d'entrevues sera identifié par un $\mathrm{E}$ suivi du numéro d'entrevue auquel il correspond.

61. Sans que la question ait été posée de manière systématique, mentionnons que plusieurs informatrices ont dit avoir utilisé la méthode Ogino-Knauss. L'une d'elles a même obtenu les informations à ce sujet par le médecin de la Goutte de lait. 
La plupart des femmes de l'échantillon ont commencé à se rendre aux Gouttes de lait dès les premiers mois de la vie de leur enfant. Certaines ont même affirmé que cette visite représentait leur première sortie quelques semaines après l'accouchement. Cinq ont décidé de se rendre à la clinique spécifiquement parce que leur bébé régurgitait constamment et maigrissait, mais, contrairement à ce qui se produisait souvent durant la première phase d'expansion, la très grande majorité y sont allées davantage à titre préventif, pour faire suivre l'enfant et s'assurer qu'il se développait normalement. De l'une à l'autre, le rythme des fréquentations varie toutefois considérablement: quelquesunes y sont allées religieusement toutes les semaines, durant plusieurs mois et ce, pour tous leurs enfants. Beaucoup s'y rendaient plutôt une à deux fois par mois durant les premiers mois, puis espaçaient les visites; une minorité a cessé d'y aller quand l'enfant avait 6 ou 8 mois seulement ${ }^{62}$. Quelques-unes se rappelaient avoir reçu la visite de l'infirmière de la Ville au moment de la naissance de leur enfant, mais, selon la plupart, il n'y avait pas véritablement de pressions pour qu'elles se présentent toutes les semaines. Elles pouvaient y aller sur une base hebdomadaire si elles le voulaient, mais le personnel des cliniques n'en faisait pas une obligation. Certaines informatrices ont quand même mentionné qu'une infirmière pouvait venir les relancer à domicile si elles s'absentaient trop longtemps: «Elles sont venues déjà à la maison pour savoir comment ça se portait là quand je n'y allais pas. Bien des fois, on ne pouvait pas y aller tout le temps, hein. C'est ça qu'il y a. Puis là bien... Oui. Ils avaient une liste là, puis ils venaient nous voir.» (E21) Selon un autre témoignage, ces visites étaient cependant très amicales et les infirmières ne faisaient pas véritablement de reproches aux femmes.

L'hiver, quand l'enfant se portait bien, plusieurs ont aussi affirmé qu'elles espaçaient les visites parce que la neige, le froid et la tâche d'habiller et de déshabiller le bébé rendaient les sorties plus difficiles. Les conditions climatiques ont donc continué d'exercer une certaine influence sur le rythme des fréquentations. Le témoignage d'une informatrice laisse voir également que le cycle des travaux ménagers pouvait être déterminant: «S'il pleuvait, bien, j'y allais un autre jour; si je trouve que ça n'adonnait pas plus que ça, si j'étais dans le grand

62. Plusieurs des informatrices avaient d'ailleurs conservé leurs «cartes bleues» (voir l'annexe I), sur lesquelles les infirmières inscrivaient la date de la visite, de même que le poids et la taille de l'enfant, et grâce auxquelles on pouvait suivre le rythme des fréquentations. Mais dans bien des cas, les informatrices ne se rappelaient pas précisément du nombre de visites qu'elles avaient effectuées. Il est donc difficile de donner plus de précisions pour l'ensemble de l'échantillon sur cette question. 
ménage, je laissais faire deux, trois semaines.» (E12) À partir du troisième et du quatrième enfant, il y avait également plus de chances qu'elles soient moins assidues, autant parce que leur travail domestique devenait plus accaparant et qu'elles avaient moins de temps à leur disposition, que parce que, après deux ou trois grossesses, elles considéraient qu'elles avaient acquis suffisamment d'expertise pour être en mesure de donner à l'enfant les soins requis: «Les deux premières, j'ai pas manqué beaucoup. Après ça, les autres, j'ai manqué un peu plus... Bien, tu te raffines un petit peu. Bien tu te dis ' $y$ 'ont mal au ventre, j'vais leur faire ci ou bien, ils ne filent pas, j'vais faire ça'.» (E1) La nécessité de faire vacciner l'enfant représentait alors la principale raison pour y retourner ${ }^{63}$.

En majorité, on peut donc dire que les femmes de l'échantillon percevaient la Goutte de lait comme un service qu'elles jugeaient important d'utiliser parce qu'il leur apparaissait comme le meilleur moyen d'assurer le bien-être de leurs enfants. Par contre, leurs visites à ces cliniques devaient leur apporter un bénéfice tangible pour qu'elles continuent de les fréquenter, et elles devaient aussi s'insérer aisément dans l'ensemble de leurs responsabilités domestiques et familiales, ce qui était beaucoup plus aisé pour celles qui avaient le moins d'enfants. En général, elles considéraient que c'était important d'y aller surtout avec leur premier bébé, alors qu'elles avaient moins d'expérience et plus de disponibilité; par la suite, tout dépendait des circonstances.

Lorsqu'on leur demandait de préciser les raisons pour lesquelles elles ont fréquenté ces cliniques, la plupart (21) ont d'abord répondu qu'elles y allaient surtout pour faire peser leur bébé. Pour plusieurs d'entre elles, en effet, la pesée représentait une grande source de motivation, sinon la raison d'être des Gouttes de lait: «...je ne serais pas allée sans ça [la pesée]. Écoute, se déplacer l'après-midi en plein hiver là, avec un carrosse ou une sleigh, c'était quelque chose, hein.» (E18) «Là, on faisait la pesée de l'enfant. Ça c'était le geste le plus important et la mensuration de l'enfant, voir s'il se développait normalement.» (E19) L'importance qu'elles attachaient à ce simple geste vient en partie du fait que, conscientes que la prise de poids représentait un des meilleurs indices pour évaluer si l'enfant se portait bien,

63. Notons qu'à partir de 1928, la Ville a entrepris d'immuniser les bébés contre la diphtérie dans les consultations municipales. Dans les années quarante, le programme de prévention contre certaines maladies comprenait la vaccination contre la variole, l'immunisation contre la diphtérie et la coqueluche et l'épreuve à la tuberculine (Sous-comité technique des services de santé, Enquête sur l'organisation du Service de santé de la Ville de Montréal (Montréal, 1948), 79). 
elles désiraient être rassurées sur les soins prodigués: «Bien ça [la pesée], c'était pour savoir 'Est-ce que je l'alimente bien, suffisamment?' 'Est-ce qu'il se développe assez bien?'. On veut toujours réussir, hein.» (E28) Le fait que le bébé avait engraissé les sécurisait, les encourageait et était même interprété comme la preuve la plus tangible de leur réussite en tant que mère: «Ils le pesaient avec la petite balance. Et quand il avait engraissé, ils nous disaient: 'Vous avez un bon bébé'. Là, toute la paroisse était fière! On avait réussi notre affaire là!» (E28) Certaines informatrices ont souligné qu'elles se rendaient compte d'elles-mêmes que le bébé «profitait bien», mais il leur semblait important d'en prendre la mesure exacte par la pesée régulière. Une donnée chiffrée, "scientifique» en quelque sorte, les satisfaisait davantage qu'une évaluation fondée sur le simple coup d'œil et plutôt approximative.

Selon les descriptions des informatrices, la visite à la Goutte de lait commençait d'ailleurs toujours par la pesée du bébé; son poids, de même que ses mensurations, étaient ensuite consignés sur une petite fiche bleue que l'infirmière remettait à la mère et si par malheur il avait maigri depuis la dernière consultation, le chiffre était inscrit en rouge afin de bien souligner qu'il s'agissait là d'une anomalie à corriger. Les concours de bébés, organisés durant cette période, étaient aussi axés sur la prise de poids régulière de l'enfant ${ }^{64}$. On peut donc dire que le personnel médical des cliniques mettait tout en œuvre pour encourager les mères à suivre de près la progression de leur enfant à ce chapitre et il n'est donc pas étonnant que les femmes aient mentionné ce facteur en si grand nombre ${ }^{65}$. Mais les commentaires entendus sur cette question démontrent également que cet aspect de la médicalisation des soins avait réussi à s'imposer. Le désir des femmes de connaître le poids exact de leur enfant témoigne en effet d'une intégration des nouvelles valeurs et attitudes que cherchaient à développer le discours médical: élever des enfants devait reposer non plus sur le sens commun, sur une approche empirique, mais se fonder sur des techniques faisant appel à la «science».

Cette insistance sur la pesée reflète par ailleurs des préoccupations plus larges à propos de l'alimentation des nourrissons. Pour diverses raisons, soit parce qu'elles considéraient qu'elles manquaient d'expé-

64. Aucune informatrice n'a mentionné ces concours comme un motif de fréquentation des Gouttes de lait. En fait, certaines ne se souvenaient pas de leur existence et seulement trois y ont participé.

65. Selon Comacchio, les cliniques pour nourrissons se transformaient souvent en «weighting station», alors que les aspects plus éducatifs étaient laissés de côté (Comacchio, Nations Are built of Babies, 123). 
rience, soit qu'elles ne voulaient pas suivre les recettes «de bonnes femmes» (un autre indice du degré de médicalisation) ou que leurs enfants digéraient mal le lait, la plupart des femmes ont dit avoir été bien contentes de recevoir des directives de la part du personnel médical à ce chapitre. De tous les conseils reçus, c'est de ceux-là dont elles avaient gardé les souvenirs les plus précis et plusieurs ont affirmé que le personnel insistait beaucoup sur l'alimentation. Le respect des consignes variait cependant énormément de l'une à l'autre. Seulement six des informatrices ont insisté pour dire qu'elles les suivaient à la lettre, ce qui pouvait d'ailleurs occasionner des désaccords avec leurs mères ou leurs belles-mères, témoignant d'une déstructuration des réseaux de transmission traditionnels des savoirs provoquée par l'intervention des experts:

[...] ma belle-mère me disait: «Donne-lui donc...». Parce qu'eux autres, quand le bébé avait trois, quatre mois, ils commençaient à lui donner des biscuits 'Village'. Ils écrasaient ça dans le lait, puis envoye donc... Alors je disais: «Non, non. Non, non. C'est ça qu'il faut que je lui donne. C'est ça qu'ils disent». Mais là, ma belle-mère ne faisait pas tout ce que je lui disais. Alors quand je partais, ils (ses beaux-parents) lui faisaient une petite bouillie [...] Non, je suivais beaucoup. C'était vraiment un peu strict, je pense. Mais tu te dis qu'un premier bébé, tu n'as pas l'expérience. Et tu penses que ça va être bien comme ça. (E18)

En général, cependant, les femmes se réservaient une marge de manœuvre beaucoup plus grande, surtout quand elles constataient que leur bébé continuait de bien se porter malgré certaines transgressions. Pour reprendre leurs expressions, elles «en prenaient et en laissaient», «passaient par dessus certaines choses» ou encore suivaient «les bons conseils» seulement, ce qui signifie qu'elles étaient prêtes à se plier aux directives lorsqu'elles-mêmes les jugeaient pertinentes ou réalistes.

Ainsi, les deux tiers des femmes de l'échantillon ont allaité, mais bien peu l'ont fait très longtemps ou avec tous leurs enfants, alors que le personnel médical insistait tout particulièrement sur ce point ${ }^{66}$. Si elles avaient plutôt tendance à préparer le lait selon les «formules» prescrites, par contre, elles ne respectaient pas nécessairement les intervalles entre les boires ou les quantités de nourriture conseillées.

66. Voir l'endos de la carte bleue, annexe I. Signalons que certaines femmes ont affirmé que leur médecin accoucheur n'était pas si enthousiaste à l'idée qu'elles allaitent et plusieurs se sont fait dire qu'elles ne pouvaient pas allaiter, car leur lait n'était pas assez abondant ou assez riche. 
Certaines, à la suggestion des femmes de leur entourage, ajoutaient du sucre à l'eau bouillie qu'elles donnaient entre les boires, alors que cette pratique était formellement condamnée ${ }^{67}$; d'autres offraient un bonbon à la menthe quand le bébé souffrait de coliques. Une informatrice a même avoué avoir endormi ses enfants en mélangeant quelques gouttes de gin à leur lait, afin de pouvoir se reposer. Lorsque le bébé pleurait sans arrêt, les femmes pouvaient donc avoir recours à différentes «recettes» et à différents «trucs», qui ne venaient pas nécessairement des médecins et des infirmières, pour les calmer. En somme, elles cherchaient plutôt à répondre aux besoins de leurs enfants par tous les moyens qu'elles connaissaient ou qu'elles avaient appris à connaître, quelle que soit leur provenance. C'est ce qui explique qu'elles affirment du même souffle avoir bien apprécié les conseils reçus en matière d'alimentation à la Goutte de lait et ne pas les avoir suivis à la lettre ou avoir même utilisé des «trucs» contreindiqués. Comme le disait une informatrice: «Vous n'aviez pas d'affaire à le dire. Vous n'étiez pas à la confesse avec eux autres.» (E12)

Si elles reconnaissaient manquer d'expérience quant à l'alimentation des bébés, par contre, la plupart des femmes ont affirmé qu'elles connaissaient déjà les mesures à prendre en ce qui concernait l'hygiène et aucune n'a mentionné avoir fréquenté les Gouttes de lait pour recevoir des conseils de cette nature. Certaines ne se souvenaient même pas d'avoir reçu des directives particulières à ce chapitre, ce qu'elles attribuaient au fait que leur bébé était propre: «Ça [l'hygiène], ils ne m'en parlaient pas bien, bien, parce qu'ils voyaient que l'enfant était tellement propre.» (E14)

$\mathrm{Si}$ on en croit leurs témoignages, toutes les femmes baignaient leur bébé quotidiennement, comme il était conseillé par le personnel médical, et la très grande majorité ont précisé qu'elles s'efforçaient de sortir tous les jours, surtout avec le premier enfant, ou bien qu'elles le laissaient dormir dans son «carrosse» à l'extérieur, quelle que soit la saison, pour «qu'il prenne l'air». Lorsque la famille s'agrandissait, il leur était cependant plus difficile de suivre ce genre de recommandation et, pour des raisons d'économies de chauffage, bien peu gardaient la fenêtre de la chambre du bébé ouverte l'hiver, comme on le suggérait à l'endos des «cartes bleues»; certaines considéraient d'ailleurs que, compte tenu du climat, ce conseil était nettement exa-

67. Mentionnons que certaines femmes ajoutaient du miel ou du sirop de maïs, ce qui était «plus naturel» selon leur expression et qu'elles disaient le faire à la suggestion de leur médecin. 
géré. Elles s'efforçaient par contre de suivre les directives concernant la stérilisation des bouteilles et des suces et le lavage des couches, même si elles ne réussissaient pas toujours ${ }^{68}$ et qu'elles reconnaissaient que ce n'était pas une mince affaire: «Ne pas trop laver [les couches] dans l'eau de Javel, les rincer beaucoup et mettre du [bi]carbonate pour adoucir l'eau. Ce n'était pas des farces, hein.» (E14) Lorsqu'elles ne le savaient pas déjà, elles appréciaient tout particulièrement recevoir des conseils économiques, comme, par exemple, d'utiliser de la fécule de maïs, plutôt que des crèmes commerciales, pour éviter les irritations. Plusieurs ont bien aimé également que l'infirmière leur montre comment repousser la peau du prépuce pour bien nettoyer le pénis de leur garçon: «Elle regardait voir, le petit garçon, si on avait bien poussé la peau du pénis. Et puis, parce qu'on a de la misère quand on a un premier enfant à faire ça, vous savez? On s'imagine qu'on va lui faire mal. Alors, avec ça, on apprend.» (E2)

De manière générale, on peut donc dire que les principes élémentaires d'hygiène étaient assez bien connus des informatrices et qu'elles étaient suffisamment conscientes de leur importance pour chercher à les respecter. Même si la moitié d'entre elles vivaient plutôt modestement, la très grande majorité, à deux exceptions près, n'ont pas connu de situation de pauvreté extrême, au point, par exemple, d'être incapables de se procurer de la glace pour conserver le lait ou du combustible pour faire bouillir de l'eau. En outre, plusieurs de ces femmes avaient des familles relativement peu nombreuses, ce qui leur laissait davantage de temps pour suivre les directives à propos de la lessive et de la stérilisation. Lorsque leurs enfants présentaient, malgré tout, des symptômes que le personnel médical associait à une mauvaise hygiène, elles avaient plutôt tendance à considérer qu'il s'agissait là de phénomènes inévitables:

Ou la fameuse, le «chapeau», la maladie qui s'appelait «faire du chapeau». [...] Fallait prendre du savon de Castille. C'était difficile à faire partir ça, ça n'avait pas de bon sens. Aujourd'hui, on n'entend plus parler de ça, je pense. On peut prévenir ça, hein? Je ne sais pas pourquoi là, mais presque tous les bébés avaient ça. On trouvait ça normal. (E19)

Selon son témoignage, cette informatrice n'a pas eu à subir les foudres de l'infirmière pour sa «négligence». En fait, trois femmes

68. Selon une informatrice, les bouteilles se brisaient lorsqu'elles les faisaient bouillir; après quelques tentatives, elle s'est contentée de les laver à l'eau savonneuse et de les rincer. 
seulement ont été réprimandées par le personnel de la Goutte de lait ${ }^{69}$. La plupart ont plutôt affirmé qu'elles étaient d'une propreté méticuleuse, que leurs enfants étaient en bonne santé et que les infirmières les avaient complimentées pour les bons soins qu'elles leur prodiguaient: «Garde Lachapelle m'a déjà dit, garde Thomas aussi, 'si toutes les mamans étaient comme vous, madame, j'vous dis qu'on aurait pas beaucoup d'ouvrage!' J'me vante là!» (E4) On peut bien sûr se demander si les informatrices ont été parfaitement honnêtes sur cette question, mais, par ailleurs, il semble évident que des femmes qui auraient été constamment rabrouées par le personnel médical n'auraient pas accepté aussi volontiers de participer à une entrevue sur ce sujet. Ajoutons que, selon leurs témoignages, très peu d'entre elles ont été témoin d'altercations entre les infirmières, les médecins et d'autres mères. Il semble bien que l'attitude générale était d'écouter attentivement les directives, sans les contester ouvertement, quitte à les ignorer une fois de retour à la maison ou à ne plus y retourner. En fait, les témoignages laissent supposer que les femmes qui fréquentaient le plus assidûment les Gouttes de lait avaient déjà adopté la plupart des principes d'hygiène que ces cliniques étaient censées leur apprendre et que ces dernières rejoignaient plus difficilement celles qui refusaient de se conformer.

La nécessité de faire vacciner les enfants a également été mentionnée par plus de la moitié des femmes pour expliquer pourquoi elles fréquentaient la Goutte de lait. À partir du deuxième ou du troisième enfant, il s'agissait même de la raison principale pour laquelle elles continuaient leurs visites, qui devenaient alors beaucoup plus espacées: «[J'y suis allée avec] le premier, le deuxième [enfant]. Après ça, je suis allée avec les autres probablement pour les vaccins. T'sais, on avait une petite carte bleue, puis tout était écrit dessus, tous les vaccins.»(E29) Selon plusieurs informatrices, le personnel médical des Gouttes de lait était assez exigeant sous ce rapport et c'est surtout lorsque venait le temps de faire vacciner leurs enfants qu'elles trouvaient important de ne pas manquer le rendez-vous qu'on leur avait fixé: «Ils étaient exigeants pour les piqûres surtout. Oui, oui. Bien oui! Beaucoup. Dans ce temps-là, il fallait... Oui. Mais ils en ont sauvé aussi beaucoup.» (E21) À la lumière des témoignages, on peut dire que l'insistance des autorités médicales sur cette question explique en bonne partie les fortes augmentations de fréquentation que

69. L'une, parce qu'elle avait donné un laxatif à son enfant au lieu d'utiliser des suppositoires; une autre, parce que sa fille, alors âgée de 8 mois, ne portait pas de chaussures; la troisième, parce qu'elle n'allaitait pas, ce qui l'a d'ailleurs décidé à abandonner la fréquentation de la clinique. 
l'on observe au tableau 3 durant les années 1950. L'idée de faire vacciner les enfants, pour mieux les protéger, semblait en effet aller de soi pour la très grande majorité des femmes interviewées et une seule informatrice a mentionné qu'elle a éprouvé certaines réticences face aux vaccins. Les progrès enregistrés quant à la mortalité infantile étaient étroitement associés à la vaccination dans l'esprit des femmes et elles ne voulaient prendre aucun risque à ce sujet. À quelques exceptions près, elles ont donc suivi scrupuleusement le programme de vaccination prescrit, jusqu'à ce que leurs enfants entrent à l'école ${ }^{70}$.

Outre la pesée et les vaccins, la gratuité du service représentait un autre incitatif majeur à fréquenter les Gouttes de lait pour plus de la moitié des informatrices. La Goutte de lait leur offrait, en effet, l'opportunité d'avoir accès à un suivi médical pour leurs enfants, que plusieurs d'entre elles n'auraient pu se payer: «J'ai aimé ça. J'ai aimé ça parce qu'on avait... parce qu'on pouvait se fier de dire qu' on avait au moins un médecin qui voyait notre bébé régulièrement... Parce qu'autrement, on aurait pas eu les moyens d'aller chez le médecin, t'sais.» (E6) Même si, en principe, les médecins ne devaient traiter que les problèmes de santé reliés à l'alimentation, bien des femmes profitaient de leurs visites pour faire examiner leurs enfants lorsqu'ils présentaient quelque symptôme anormal: «T'sais, des fois si je m'apercevais que les enfants avaient mal aux oreilles ou qu'il y avait quelque chose qui ne marchait pas, bien t'allais à la Goutte de lait. Puis ça ne te coûtait rien. Tandis que t'allais chez ton médecin bien, tu payais. C'était pas gros une piastre. Mais dans ce temps-là, une piastre, c'était une piastre.» (E1) En outre, selon certains témoignages, les Gouttes de lait distribuaient gratuitement des échantillons de vitamines ou de compléments alimentaires, comme de l'huile de foie de morue, de l'Ostoco et de l'Infantol, ou fournissaient certains médicaments, comme les gouttes pour les yeux et les oreilles, ce qui était apprécié des femmes: "C'était gratuit. Comme l'huile de foie de morue, ils nous le donnaient, avec de la vitamine $\mathrm{D}$ dedans, exprès pour donner aux enfants. Et puis, ensuite, ils nous donnaient de l'Infantol. C'était des échantillons qu'ils avaient mais qu'importe, ils nous le donnaient quand même hein?» (E4) Il faut préciser que les informatrices qui ont invoqué ce motif n'étaient pas nécessairement parmi les plus pauvres de l'échantillon, ce qui montre bien que les frais médicaux représentaient une dépense assez considérable, même dans un contexte de plus grande prospérité économique, comme ce fut le

70. À partir de la fin des années trente, le Service de santé de la Ville inaugure des consultations pour les enfants d'âge pré-scolaire qui sont jumelées aux consultations pour nourrissons et où les 2 à 5 ans reçoivent les rappels de vaccins. 
cas durant les années 1940 et 1950 . Plusieurs d'entre elles ont mentionné qu'elles avaient un médecin de famille, très souvent celui qui les avait accouchées, mais selon la plupart, il n'était pas dans leurs habitudes de l'appeler au moindre symptôme parce qu'il fallait payer. Ces femmes essayaient donc de tirer partie des ressources qui s'offraient à elles afin d'assurer un suivi médical à leurs enfants, sans y consacrer une part trop grande du budget familial.

La pesée, les conseils à propos de l'alimentation, les vaccins et la gratuité des services médicaux représentaient donc, pour ces femmes, des sources importantes de motivations pour fréquenter les Gouttes de lait. C'est donc dire qu'elles avaient intégré l'idée de s'en remettre, jusqu'à un certain point, à des spécialistes pour prévenir la maladie et conserver la santé de leurs enfants, ce que la Goutte de lait leur permettait de faire sans engloutir des sommes d'argent importantes. La baisse de la mortalité infantile, qui commençait à devenir manifeste durant les années 1930 et qui s'est accentuée par la suite, était certainement de nature à les convaincre de l'impact positif de ce type de service. Leur assiduité, on l'a vu, n'était pas parfaite et variait en fonction d'impératifs d'ordre domestique ou du nombre d'enfants qu'elles avaient à leur charge et elles ne suivaient pas nécessairement toutes les directives qu'on leur donnait, mais la plupart considéraient qu'elles ne pouvaient complètement faire abstraction de l'existence de ces cliniques, n'était-ce que pour se faire confirmer qu'elles donnaient de bons soins à leurs enfants et pour le programme de vaccination. Il faut dire aussi que les deux tiers de ces femmes ont vu régulièrement un médecin durant leurs grossesses, ce qui signifie qu'avant même la naissance de l'enfant, elles avaient pris l'habitude de consulter un praticien. Parmi celles qui ne l'ont pas fait, on retrouve les femmes les plus pauvres de l'échantillon, et souvent celles qui ont fréquenté les Gouttes de lait avec le moins d'assiduité. Une corrélation semble donc se dégager entre le niveau de vie, le fait de consulter régulièrement un médecin durant la grossesse et la fréquentation de ces cliniques.

Mais les femmes allaient aussi à la Goutte de lait pour des raisons tout à fait étrangères à ces questions purement médicales. Pour les deux tiers des femmes interviewées, ces visites représentaient également une sortie et même une occasion de rencontrer d'autres femmes. Plusieurs d'entre elles ont précisé qu'elles n'avaient pas souvent l'occasion de quitter la maison ou de voir d'autres personnes en dehors de la famille, et que les visites à la clinique leur permettaient de faire des rencontres agréables: «Ça fait une place où aller puis ça changeait les idées de la maison, du quotidien. [...] On jasait. Puis ça faisait notre affaire, nous autres. Parce que, avec des enfants, 
on avait pas de grandes occasions, vous savez, de jaser, hein, puis de rencontrer des gens dans ce temps-là, là.» (E14) Selon une informatrice, c'était même l'aspect de la visite qui était le plus intéressant: «C'était l'fun. Ah! Mon Dieu. C'était l'fun. Ça, je pense, que c'était l'affaire qu'on aimait le plus sans le savoir [sans se l'avouer?].» (E28) Pendant qu'elles attendaient pour voir l'infirmière ou le médecin, les femmes parlaient évidemment de leurs bébés; elles s'encourageaient, se racontaient leurs difficultés ou leurs réussites, admiraient mutuellement leurs enfants et échangeaient même des conseils, une pratique que les médecins de l'époque cherchaient justement à éviter: «Bon, on se transmettait des conseils. On se disait: 'Comment ça va? Qu'est-ce que vous faites donc?' On se donnait des trucs. Puis c'est comme ça que je me suis débrouillée pas mal.» (E11) Pour plusieurs informatrices, fréquenter les Gouttes de lait était donc aussi un moyen de briser l'isolement et de vivre des moments de solidarité avec d'autres femmes. Comme certaines l'ont fait remarquer, elles étaient toutes «dans le même bateau» du fait qu'elles étaient de jeunes mères: «C'était agréable. [...] Parce que disons qu'on rencontre, surtout pour ton premier bébé là, on rencontrait d'autres personnes qui étaient... comme nous autres.» (E18) Côtoyer des femmes aux prises avec les mêmes problèmes, les mêmes inquiétudes, représentait une forme de support, une façon de se rassurer, tout comme le fait, d'ailleurs, de rencontrer le médecin ou l'infirmière:

T'sais, on en arrachait toutes. Parce que, t'sais, sur la rue Bordeaux dans le bas de la ville, je te dis que ce n'était pas riche dans ce temps-là. Alors on était toutes dans la même situation, hein. Alors on s'encourageait... Puis quand on partait de là, on était bien contente. T'sais, on était satisfaite parce qu'on ne se sentait pas toute seule, $t$ 'sais. Parce que si il y avait des petites affaires de croches, bien ils nous le disaient. Ils nous encourageaient. (E21)

Ces rencontres étaient également une occasion pour les femmes de se toiletter mais, surtout, d'exhiber fièrement leurs enfants dans leurs plus beaux atours: «Puis là, tu tricotais un petit gilet à ton bébé puis t'étais contente d'aller le montrer.» (E1) Une informatrice, mère de 11 enfants, confiait même qu'elle réservait certains vêtements pour ce genre d'occasion, afin de cacher sa pauvreté:

C'était une sortie. [...] On pouvait s'habiller en propre. Et puis là on portait un chapeau dans ce temps-là, puis tout ça. Moi, j'adorais ça. Et je gardais toujours, moi, des vêtements pour mes enfants [...], seulement pour sortir. Là c'étaient des beaux vêtements qui restaient propres. Puis là, ça me faisait une fierté de 
bien les habiller pour ne pas que ça paraisse qu'on était pauvre. Parce que on était pauvre, mais pas au point de manquer de manger là. [...] Mais je ne voulais pas que ça paraisse, là t'sé. On ne pouvait pas dire, là t'sé. Ils avaient des vêtements qui servaient, là, juste pour ça. Puis on sortait ces vêtements-là puis c'était pour sortir. Ça ne paraissait pas qu'on était classe ouvrière. (E19)

Lors de leurs visites à la Goutte de lait, les mères avaient donc à cœur de présenter leurs enfants sous leur meilleur jour. Un bébé bien habillé, mais aussi bien propre et respirant la santé, était une preuve de leur compétence et de leur réussite en tant que mère: «Puis on aurait dit que les femmes étaient contentes, $t$ 'sais, de venir montrer leurs bébés, puis de... Ah, ça [se] faisait une gloire puis un honneur.» (E1) Ces témoignages, comme bien d'autres, laissent voir que la maternité représentait pour bien des femmes une part importante de leur vie, un accomplissement qu'elles cherchaient à faire reconnaître par leurs pairs et par le personnel médical. Les félicitations ou les compliments qu'elles recevaient à propos de leurs enfants étaient perçus comme une reconnaissance de leur qualité de mère. Dans le contexte des années 1940 et 1950 en particulier, alors que les valeurs familiales ont été plus que jamais à l'avant-scène, que les conditions économiques se sont améliorées pour une bonne proportion de la population et que l'idéal du couple pourvoyeur-ménagère devenait plus que jamais accessible, ces femmes trouvaient une source importante de valorisation dans les bons soins qu'elles prodiguaient à leurs enfants, et la Goutte de lait leur donnait l'occasion d'étaler publiquement leur réussite ${ }^{71}$.

Cet orgueil que les mères éprouvaient envers leur bébé avait aussi son revers. Ainsi, une informatrice n'a pas consulté les Gouttes de lait avec l'un de ses enfants parce qu'il était né avec une difformité et qu'elle ne voulait pas l'exposer aux regards et à la curiosité des autres femmes du voisinage. Deux autres, les plus pauvres de l'échantillon,

71. Sur le niveau de vie de la classe ouvrière durant cette période, voir Jean-Pierre Charland, Système technique et bonheur domestique. Rémunération, consommation et pauvreté au Québec, 1920-1960 (Québec, Institut québécois de recherche sur la culture, 1992); Simon Langlois, "L'avènement de la société de consommation: un tournant dans l'histoire de la famille», dans Denise Lemieux, Familles d'aujourd'hui (Québec, Institut québécois de recherche sur la culture, 1990), 89-43. Sur les idéologies entourant la famille durant les années cinquante, voir, entre autres, Veronica Strong-Boag, «Home Dreams: Women and the Suburban Experiment in Canada», Canadian Historical Review, 72,4 (1991): 471-503; le Collectif Clio, Histoire des femmes au Québec depuis quatre siècles (Montréal, Le Jour, 1992), 413 et ss. Sur le contexte américain, voir Elaine Tyler May, Homeward Bound: American Families in the Cold War Era (New York, Basic Books, 1988). 
y sont allées à quelques reprises seulement. Par ses commentaires, une informatrice démontrait d'ailleurs que la compétition subtile que se livraient les mères à propos des enfants pouvait déboucher sur une forme d'ostracisme: «Et puis quand il y en avait une que son enfant sentait un petit peu le «sûr»... Ah! Ça regardait, t'sais en voulant dire on ne s'assoit pas proche d'elle.» (E28) En même temps qu'elles devenaient pour certaines femmes un lieu de socialisation et d'échanges, un endroit où faire étalage de sa réussite et de sa fierté maternelle, les cliniques pouvaient donc aussi apparaître bien inhospitalières à celles qui n'arrivaient pas à soutenir la comparaison.

Plus des deux tiers des femmes de l'échantillon évaluaient de façon très positive leur expérience de la Goutte de lait. D'ailleurs, plusieurs ont affirmé avoir accepté d'être interviewées parce qu'elles en gardaient un bon souvenir et l'une d'elles a même précisé qu'elle avait voulu, de cette manière, remercier le personnel. Leurs témoignages démontrent que ce qu'elles ont le plus apprécié, c'est le travail des infirmières, avec lesquelles d'ailleurs elles étaient le plus en contact. Dans plusieurs cliniques, effectivement, les femmes ne voyaient pas le médecin à chacune de leurs visites. Quand l'enfant se portait bien, la consultation avec le praticien était laissée à leur discrétion et bien des femmes préféraient ne pas le voir: «j'étais contente de ce qu'elle [l'infirmière] me disait parce que ça réussissait. [...] Si l'enfant était malade, on y allait [voir le médecin]. Mais s'il n'était pas malade... le reste, ça ne le regardait pas, lui. Lui, c'était les malades.» (E23) Le personnage de l'infirmière prenait donc davantage de place dans leurs souvenirs et, bien souvent, elles se rappelaient de leurs noms et ne tarissaient pas d'éloges à leur sujet: "Y'étaient bien fines les gardes-malades. Celles qu'on a eues, nous autres, en tou'cas...» (E4); «Ah Mon Dieu! C'était dévouée, ça, dévouée! C'était effrayant! Il me semble de la voir. Elle était «bacquesse». Je te dis qu'elle y allait par là puis, t'sais, c'était pas drôle. Elle était fine, fine. Oui. ${ }^{72}$ » (E15) C'est aussi à l'infirmière que l'on se confiait le plus et à qui l'on demandait le plus de conseils. Dans beaucoup de récits, le médecin joue un rôle plus effacé; il semble plus distant, moins familier. En raison de son sexe, l'infirmière semblait d'emblée plus douce, patiente, dévouée et les femmes avaient l'impression qu'elles les comprenaient davantage: «[Je posais plus de questions] à l'infirmière. Moi, les médecins... J'ai rien contre les médecins, [mais] c'est des hommes. J'suis gênée avec... Puis ils nous comprennent pas. Peut-être

72. Une autre informatrice a aussi connu cette infirmière dont elle parlait dans des termes très élogieux car elle avait détecté un problème cardiaque chez sa fille: «Ils faisaient tout un examen. Et puis c'est garde Lachapelle qui a vu que ma fille [...] avait un défaut au cœur» (E4). 
que c'est pas vrai, mais en tout cas.» (E3) En fait, les témoignages démontrent que les infirmières jouaient un rôle central dans les Gouttes de lait et qu'elles transmettaient la plupart des conseils et des directives. Elles inspiraient confiance et on peut affirmer que, sans leur présence, les cliniques n'auraient pas rejoint autant de femmes ${ }^{73}$. C'est donc dire également que le processus de «médicalisation» des soins de la petite enfance a été médiatisé par ce groupe de professionnelles de la santé et que, s'il faut parler d'un contrôle médical sur les mères, il faut bien reconnaître qu'une large part de ce contrôle était exercé par d'autres femmes.

\section{CONCLUSION}

À l'aide de sources d'origines très différentes, nous avons tenté de cerner la fréquentation des Gouttes de lait montréalaises, depuis leurs premières années d'existence jusqu'à une période relativement récente. Chacune de ces sources comporte bien sûr ses limites, mais, dans une certaine mesure, elles se complètent et permettent de dégager certaines conclusions au regard des motifs qui amenaient les femmes à fréquenter, ou non, ces cliniques.

D'une part, il semble évident que la très grande pauvreté représentait une véritable entrave à la fréquentation des Gouttes de lait. Les rapports de la Commission centrale de la FNSJB, de même que certaines des entrevues, confirment que, pour se prévaloir de leurs services, surtout sur une base régulière, il fallait bénéficier d'un niveau de vie minimal, permettant au moins à la mère et aux enfants d'être habillés convenablement. L'extrême dénuement et même la pauvreté relative vécue dans un contexte de prospérité, comme ce fut le cas dans les années 1940 et 1950 , n'étaient pas de nature à susciter le recours à ces cliniques, alors même que les enfants provenant de ces familles étaient les plus à risques en ce qui concerne la morbidité et la mortalité. Il est également possible que l'abstention d'une partie des mères, souvent les plus pauvres, reflète une impossibilité, voire un refus, d'adopter les nouvelles normes en matière de soins aux enfants parce qu'elles s'écartaient trop radicalement des habitudes en usage dans leur milieu. Quoi qu'il en soit, on peut dire que les Gouttes de lait ont raté une partie importante de leur cible et que le déclin de la

73. Les promoteurs de la santé publique étaient d'ailleurs bien conscients de ce fait. Voir à ce sujet Meryn Stuart, «Shifting Professional Boundaries: Gender Conflict in Public Health, 1920-1925», dans Dianne Dodd et Deborah Gorham, dir., Caring and Curing. Historical Perspectives on Women and Healing in Canada (Ottawa, Presses de l'Université d'Ottawa, 1994), 49-70. 
mortalité infantile ne peut être attribué entièrement à leur seule présence.

D'autre part, les entrevues réalisées, de même que les statistiques du Service de santé de la Ville de Montréal, ont permis de constater que la fréquentation des Gouttes de lait au cours de la période 19351965 était davantage ancrée dans les mœurs. Le message hygiéniste avait fait son chemin au sein de la population féminine et les femmes désiraient s'en remettre à des spécialistes en puériculture, médecins ou infirmières, du moins lors des premières naissances, pour s'assurer du bon développement physique de leur enfant, obtenir des conseils, de même que certains services médicaux, comme les vaccins. La baisse de la mortalité infantile, qui s'est accentuée au cours des années 1930 et 1940 , n'est pas étrangère à ce phénomène. Parce que la santé et la vie des nourrissons pouvaient désormais être préservées dans des proportions beaucoup plus grandes, en partie sans doute grâce à la fréquentation des Gouttes de lait et à la diffusion du message hygiéniste, elles sont devenues l'objet de préoccupations plus larges de la part des mères. Celles-ci ont alors plus volontiers accepté d'utiliser un service qui leur donnait gratuitement accès à une expertise médicale jugée responsable de ces améliorations. Ce n'est d'ailleurs pas seulement pour les soins infantiles que les femmes sollicitaient l'intervention des médecins, comme en témoigne la multiplication des consultations au moment de leurs grossesses. Au cours de ces années qui ont été témoins de percées scientifiques et technologiques importantes (on pense par exemple aux sulfamides et aux antibiotiques), les risques associés à l'enfantement et à l'enfance sont devenus moins tolérables et leur médicalisation plus acceptable. Les témoignages montrent toutefois que certaines conditions devaient être réunies pour que les femmes acceptent de participer à ce processus de médicalisation. La diminution de la taille de la famille et l'espacement des naissances, des choix faits par de plus en plus de couples à partir des années 1930, ont très largement favorisé la fréquentation régulière des cliniques. Moins accaparées par leurs tâches domestiques, les femmes pouvaient alors plus difficilement choisir d'ignorer complètement un service présenté comme essentiel au bien-être et même à la survie de leurs enfants. C'est donc à partir du moment où elles ont commencé à limiter leur fécondité que les médecins ont pu les amener à inclure la visite régulière de la Goutte de lait parmi leurs responsabilités maternelles.

Par ailleurs, aller à la Goutte de lait ne signifiait pas que les femmes s'en remettaient uniquement aux médecins ou aux infirmières pour décider de ce qui convenait à leurs bébés. Même si, par exemple, 
bien des femmes ne voulaient pas utiliser des recettes de «grandmère» pour nourrir leurs enfants, indice d'une intégration des préceptes scientifiques, elles faisaient également appel aux conseils de leur entourage ou elles s'en remettaient à leur propre jugement. Le personnel médical de la Goutte de lait représentait donc une ressource importante pour les aider à déterminer le type de régime nécessaire à leurs bébés mais pas nécessairement une source exclusive d'information.

Enfin, loin d'être une corvée, les visites à la Goutte de lait étaient perçues par une majorité des informatrices comme une occasion de socialiser avec d'autres femmes et d'exhiber fièrement leur progéniture. À ce chapitre, les témoignages démontrent qu'elles allaient chercher dans ces cliniques non seulement des conseils ou des services médicaux, mais aussi une forme de solidarité et une reconnaissance sociale de leur dévouement maternel. L'intervention de l'État et des professionnels de la santé dans le domaine des soins aux enfants, si elle peut être considérée comme une intrusion dans la sphère privée ou comme une forme de régulation sociale, faisait donc aussi en sorte de créer un espace public où les mères pouvaient se rencontrer, échanger et faire valoir leur compétence maternelle. En confiant de nouvelles responsabilités aux mères par rapport à la santé de leurs enfants, le discours médical leur offrait aussi une base supplémentaire de fierté et de valorisation que les femmes n'ont pas été longues à saisir. En retour, cette valorisation ne pouvait que les encourager à s'identifier étroitement à leur rôle de mère, une dimension qui ressort aussi nettement des témoignages. 


\section{ANNEXE I \\ Endos de la carte bleue des Gouttes de lait}

\section{7}

\section{Conseils pratiques}

$1^{\circ}$ Une mère ne doit jamais pouvoir se reprocher de n'avoir pas tout fait pour allaiter elle-même son enfant.

$2^{\circ}$ L'enfant a droit au lait de sa mère, car rien ne saurait remplacer avantageusement ce lait au cours de la première année.

$3^{\circ}$ Que l'enfant soit allaité par sa mère ou qu'il reçoive un autre lait, les repas doivent être donnés à intervalles réguliers.

$4^{0}$ À moins d'avis contraire de votre médecin, ces repas, dès la naissance, seront donnés toutes les trois heures le jour, et une fois la nuit, soit sept repas en vingt-quatre heures. Après six mois le repas de la nuit doit être supprimé.

$5^{\circ}$ Durant la première année, l'enfant ne doit prendre que du lait. Les autres nourritures telles que bouillies, soupes, sauces, panades, patates, etc., doivent être considérées comme dangereuses, et n'être données, dans certains cas particuliers, que sur avis de votre médecin.

$6^{\circ} \mathrm{Si}$ votre enfant crie dans l'intervalle des repas, donnez-lui un peu d'eau bouillie non sucrée.

$7^{\circ} \mathrm{Si}$ après l'avoir soumis à un régime bien ordonné, votre enfant continue à crier, dort peu, digère mal, vomit, passe des selles qui ne sont pas naturelles, consultez votre médecin ou venez à la consultation.

$8^{\circ} \mathrm{Si}$ votre enfant est pris de diarrhée, supprimez toute nourriture, donnez une cuillérée à thé d'huile de ricin, et de l'eau bouillie seulement, en attendant la consultation.

$9^{\circ} \mathrm{Ne}$ donnez jamais aucun remède sans l'avis du médecin.

$10^{\circ} \mathrm{Ne}$ donnez jamais aucun sirop calmant.

$11^{\circ} \mathrm{La}$ balance est le meilleur guide pour juger du développement de votre enfant. Pesez-le toujours au moins une fois par semaine.

$12^{\circ}$ Baignez votre enfant tous les jours. S'il dort mal, répétez ce bain au moment du coucher.

$13^{\circ} \mathrm{L}$ 'enfant a besoin de soleil et d'air pur. Sortez-le tous les jours. Laissez la fenêtre entr'ouverte en toute saison. Si vous craignez qu'il ne se refroidisse, couvrez-le plus chaudement. 


\section{4}

Conseils pratiques

$1^{\circ}$ Une mère doit ALLAITER SON BÉBÉ.

$2^{\circ}$ L'enfant a droit au lait de sa mère, CAR RIEN NE SAURAIT REMPLACER AVANTAGEUSEMENT CE LAIT au cours de la première année.

$3^{\circ}$ Que le bébé soit allaité par sa mère ou qu'il reçoive un autre lait, les repas doivent être DONNÉS À INTERVALLES RÉGULIERS.

$4^{\text {o }}$ À moins d'avis contraire de votre médecin, ces repas, dès la naissance, seront donnés toutes les trois ou quatre heures le jour, et une fois la nuit, soit six ou sept repas en vingt-quatre heures. Après trois mois, le repas de la nuit doit être supprimé.

$5^{\circ} \mathrm{Si}$ votre enfant crie dans l'intervalle des repas, donnez-lui un peu d'eau bouillie non sucrée.

$6^{\circ} \mathrm{Si}$ après l'avoir soumis à un régime bien ordonné, votre enfant crie encore, s'il dort peu, s'il digère mal, s'il vomit, s'il a des selles anormales, CONSULTEZ VOTRE MÉDECIN OU VENEZ À LA CONSULTATION.

$7^{\circ} \mathrm{Ne}$ donnez jamais de sirop calmant, ne donnez jamais de remèdes, SANS L'AVIS DU MÉDECIN.

$8^{\circ} \mathrm{La}$ balance est le meilleur guide pour juger du développement de votre enfant. PESEZ-LE TOUJOURS AU MOINS UNE FOIS PAR SEMAINE.

$9^{\circ}$ Baignez votre bébé tous les jours. S'il dort mal, répétez ce bain au moment du coucher.

$10^{\circ}$ L'enfant a besoin de sommeil et d'air pur. Promenez-le tous les jours. Laissez la fenêtre entr'ouverte en toute saison. SI VOUS CRAIGNEZ QU'IL NE SE REFROIDISSE, COUVREZ-LE PLUS CHAUDEMENT. 